\title{
LARGE SOLUTIONS TO AN ANISOTROPIC QUASILINEAR ELLIPTIC PROBLEM
}

\author{
JORGE GARCÍA-MELIÁN, JULIO D. ROSSI AND JOSÉ C. SABINA DE LIS
}

\begin{abstract}
In this paper we consider existence, asymptotic behavior near the boundary and uniqueness of positive solutions to the problem$$
\operatorname{div}_{x}\left(\left|\nabla_{x} u\right|^{p-2} \nabla_{x} u\right)(x, y)+\operatorname{div}_{y}\left(\left|\nabla_{y} u\right|^{q-2} \nabla_{y} u\right)(x, y)=u^{r}(x, y)
$$

in a bounded domain $\Omega \subset \mathbb{R}^{N} \times \mathbb{R}^{M}$, together with the boundary condition $u(x, y)=\infty$ on $\partial \Omega$. We prove that the necessary and sufficient condition for the existence of a solution $u \in W_{l o c}^{1, p, q}(\Omega)$ to this problem is $r>\max \{p-1, q-1\}$. Assuming that $r>q-1 \geq p-1>0$ we will show that the exponent $q$ controls the blow-up rates near the boundary in the sense that all points of $\partial \Omega$ share the same profile, that depends on $q$ and $r$ but not on $p$, with the sole exception of the vertical points (where the exponent $p$ plays a role).
\end{abstract}

\section{INTRODUCTION}

We will be concerned in this paper with the study of positive solutions to the problem

$$
\begin{cases}\Delta_{p, q} u(x, y)=u^{r}(x, y) & \text { in } \Omega, \\ u=+\infty & \text { on } \partial \Omega\end{cases}
$$

where $\Omega$ is a smooth bounded domain in $\mathbb{R}^{N} \times \mathbb{R}^{M}, N, M \geq 1$ and $\Delta_{p, q} u$ stands for the combination $\operatorname{div}_{x}\left(\left|\nabla_{x} u\right|^{p-2} \nabla_{x} u\right)+\operatorname{div}_{y}\left(\left|\nabla_{y} u\right|^{q-2} \nabla_{y} u\right)$, of the standard $p$ and $q$-Laplacian operators with $p, q>1$ acting separately in each group of variables $x \in \mathbb{R}^{N}$ and $y \in \mathbb{R}^{M}$. This definition of $\Delta_{p, q} u$ is understood in a distributional sense in a suitable subspace of $W_{l o c}^{1, p \wedge q}(\Omega)$ with $p \wedge q=\min \{p, q\}$. The boundary condition is understood in the sense that $u(x, y) \rightarrow \infty$ as $(x, y) \rightarrow \partial \Omega$. Solutions to problem (1.1) will be regarded in a weak sense concerning both the equation and the boundary condition (see Section 2 for a precise statement). Without loss of generality it will be assumed in the sequel that $1<p \leq q$.

Problems like (1.1) which involve a singular Dirichlet condition on $\partial \Omega$ are usually known in the literature as "boundary blow-up problems", and their solutions are also termed "large solutions". Since the pioneering works of Bieberbach, [5], and Rademacher, 27], and further continuations by Keller, [18], Osserman, [26], Loewner-Nirenberg, [23], and Bandle-Marcus [2], a great amount of research has been devoted to study such problems. In fact, they arise in completely different fields as Riemannian geometry or population dynamics. We refer the interested reader to [12] and [28] for a complete updated account of references and applications. 
In most of the cases treated in the literature, the operator considered in (1.1) is the linear 2-Laplacian $\Delta u=\Delta_{x} u+\Delta_{y} u=f(u)(p=q=2)$ with different kinds of nonlinearities as reaction terms. Of particular importance are the special cases $f(u)=u^{r}$ and $f(u)=e^{u}$. On the other hand, only few works have dealt with the $p$-Laplacian operator as left hand side in (1.1) (see [8], 25], [13, 14] and Remark 2 below).

As for the operator in (1.1) its analysis belongs to the realm of anisotropic elliptic equations also known as elliptic equations under non standard (or "non natural") growth conditions. Such area has deserved a great deal of interest since the last eighties, specially regarding the regularity issues. See [16], 24], [22] to quote only a few early works on the subject and [11, [9], [10], 32] and 34 for additional recent literature on operators exhibiting anisotropic growth in the gradient that are complemented with standard (finite) boundary conditions. Concerning physical models involving this kind of operators we refer to the recent survey [1] (see also [3, [4] for further models in mathematical biology involving operators as the one considered in (1.1)). On the other hand, we should emphasize that, at the best of our knowledge, this is the first time where a quasilinear operator involving a different growth rate in the gradient with respect to separate groups of variables is considered when dealing with large solutions.

Our main result here can be summarized as follows: A necessary and sufficient condition for the existence of a solution $u \in W_{l o c}^{1, p, q}(\Omega)$ to the problem (1.1) is

$$
r>\max \{p-1, q-1\} .
$$

Provided that such condition holds then (1.1) possesses a minimal positive solution $u \in W_{l o c}^{1, p, q}(\Omega) \cap L_{l o c}^{\infty}(\Omega)$ such that $v(x) \leq u(x), x \in \Omega$, for every solution $v \in W^{1, p, q}(\Omega)$ to the equation $\Delta_{p, q} u=u^{r}$ in $\Omega$ with finite boundary datum (see next section for the definition of the space $W_{l o c}^{1, p, q}(\Omega)$ ). In addition, the positive solution is unique if the domain $\Omega$ verifies a certain "starshapedness" condition.

Since, due to the boundary condition in (1.1), solutions satisfy $u(x, y) \rightarrow$ $\infty$ as $(x, y) \rightarrow \partial \Omega$, we also analyze their asymptotic behavior near the boundary (the speed at which solutions go to infinity when $(x, y) \rightarrow \partial \Omega$ ). Assuming that $q>p$ we will show that the variable $y$ controls the blow-up rates in the sense that all points of $\partial \Omega$ share the same profile -in order of magnitude- as the horizontal points, with the sole exception of the vertical points (see Section 4 for a precise definition of horizontal and vertical points). Furthermore, we also prove that the upper estimate at vertical points $z_{0}$ strongly depends on the shape of the boundary $\partial \Omega$ in a neighborhood of $z_{0}$, more precisely, on the order of tangency of $\partial \Omega$ in the $y$-direction (see also Section 4 for a precise definition of order of tangency). Of course, symmetric results would be obtained by properly reversing the rôles of the variables $x$ and $y$ if $p>q$.

All these features clearly reflect the strong anisotropic character of our problem. 
As a previous step for our proofs we look in detail at the simpler case in which the domain is a product $\Omega=\Omega_{1} \times \Omega_{2}$ with special emphasis in the cases $\Omega=B_{1} \times B_{2}$ (the product of two balls) and $\Omega=\mathbb{R}_{+}^{N} \times \mathbb{R}_{+}^{M}$ (the product of two half-spaces).

The paper is organized as follows: in Section 2 we collect some preliminary results which are mainly concerned with problem (1.1) with a finite datum on $\partial \Omega$; in Section 3 we consider the case in which we can use a separation of variables, that is when the domain is of the form $\Omega=\Omega_{1} \times \Omega_{2}$. Section 4 is devoted to consider the issues of existence and uniqueness in general domains, while the boundary behavior of solutions in this general case is contained in Section 5 .

\section{Preliminary Results}

In this section we collect some preliminary results which mainly deal with problem (1.1) with a finite datum as a boundary condition.

Let $\Omega \subset \mathbb{R}^{N} \times \mathbb{R}^{M}$ be an open set. For functions defined in $\Omega$ we use the notation $u(x, y)$, where $x \in \mathbb{R}^{N}, y \in \mathbb{R}^{M},(x, y) \in \Omega$. Let us introduce the space,

$$
\begin{aligned}
& W^{1, p, q}(\Omega)= \\
& \quad\left\{u \in L^{p}(\Omega): \frac{\partial u}{\partial x_{i}} \in L^{p}(\Omega), \frac{\partial u}{\partial y_{j}} \in L^{q}(\Omega), 1 \leq i \leq N, 1 \leq j \leq M\right\},
\end{aligned}
$$

where derivatives are being considered in the weak sense. It is clear that $W^{1, p, q}(\Omega)$ is a Banach space when endowed with the norm

$$
\|u\|:=|u|_{p}+\left|\nabla_{x} u\right|_{p}+\left|\nabla_{y} u\right|_{q},
$$

that is in addition reflexive provided $1<p \leq q<\infty$. In the literature, $W^{1, p, q}(\Omega)$ is often referred to as an "anisotropic" Sobolev space (see [29], [30]). As customary, $W_{0}^{1, p, q}(\Omega)$ will designate the completion of $C_{0}^{\infty}(\Omega)$ in $W^{1, p, q}(\Omega)$, while the space $W_{l o c}^{1, p, q}(\Omega)$ comprises all those measurable functions $u$ in $\Omega$ such that $u \in W^{1, p, q}(Q)$ for each bounded open set $Q$ such that $\bar{Q} \subset \Omega$. Since $W^{1, p, q}(\Omega) \subset W^{1, p}(\Omega)$ (recall that we are assuming $1<p \leq q$ ), restrictions of functions $u \in W^{1, p, q}(\Omega)$ to the boundary $\partial \Omega$ will be considered in the sense of traces. Thus, $u_{\mid \partial \Omega} \in W^{1-1 / p, p}(\partial \Omega)$ if $u \in W^{1, p, q}(\Omega)$.

Let us state next what we will understand as a solution to (1.1).

Definition. A nonnegative function $u \in W_{l o c}^{1, p, q}(\Omega) \cap L_{l o c}^{\infty}(\Omega)$ is called a (weak) solution to (1.1) if

$$
\int_{\Omega}\left|\nabla_{x} u\right|^{p-2} \nabla_{x} u \nabla_{x} \varphi+\left|\nabla_{y} u\right|^{q-2} \nabla_{y} u \nabla_{y} \varphi+\int_{\Omega} u^{r} \varphi=0,
$$

for all $\varphi \in W^{1, p, q}(\Omega)$, compactly supported in $\Omega$. In addition, the boundary condition is understood in the sense that $(k-u)^{+} \in W_{0}^{1, p, q}(\Omega)$ for arbitrary $k>0$. 
For future use in this work, $u \geq 0$ on $\partial \Omega\left(u \in W^{1, p, q}(\Omega)\right)$ will mean $u^{-} \in W_{0}^{1, p, q}(\Omega)$ (see [17]). Comparison between functions of $W^{1, p, q}(\Omega)$ on $\partial \Omega$ will always be understood in this sense.

To show the existence of solutions to (1.1), we first analyze the finite boundary value problem, namely

$$
\begin{cases}\Delta_{p, q} u=|u|^{r-1} u & \text { in } \Omega, \\ u=g & \text { on } \partial \Omega .\end{cases}
$$

For $g \in W^{1, p, q}(\Omega)$, a solution $u \in W^{1, p, q}(\Omega) \cap L^{p^{\prime} r}(\Omega)$ to $(2.2)$ is defined through the equality (2.1) by employing test functions $\varphi \in W_{0}^{1, p, q}(\Omega)$ and requiring that $u-g \in W_{0}^{1, p, q}(\Omega)$. Subsolutions $\underline{u} \in W^{1, p, q}(\Omega) \cap L^{p^{\prime} r}(\Omega)$ are defined by replacing equality by $\leq$ in $(2.1)$, using nonnegative test functions $\varphi$ and requiring that $(\underline{u}-g)^{+} \in W_{0}^{1, p, q}(\Omega)$, supersolutions being defined by properly reversing the inequalities. Since we will be henceforth interested in positive solutions, we are assuming $g \geq 0$, and thus the absolute value in the right-hand side of $(2.2)$ can be dropped.

We start by stating a comparison result, which in particular implies uniqueness of positive solutions to (2.2).

Lemma 1. Let $\underline{u}, \bar{u} \in W^{1, p, q}(\Omega) \cap L^{p^{\prime} r}(\Omega)$ be nonnegative subsolution and supersolution, respectively, of the equation in (2.2) with $\underline{u} \leq \bar{u}$ on $\partial \Omega$. Then $\underline{u} \leq \bar{u}$ a.e. in $\Omega$.

Proof. Using the same (nonnegative) test function $\varphi \in W_{0}^{1, p, q}(\Omega)$ in the inequalities for $\underline{u}$ and $\bar{u}$ we get, after substraction

$$
\begin{aligned}
& \int_{\Omega}\left\{\left(\left|\nabla_{x} \underline{u}\right|^{p-2} \nabla_{x} \underline{u}-\left|\nabla_{x} \bar{u}\right|^{p-2} \nabla_{x} \bar{u}\right) \nabla_{x} \varphi\right\} \\
& \quad+\int_{\Omega}\left\{\left(\left|\nabla_{y} \underline{u}\right|^{q-2} \nabla_{y} \underline{u}-\left|\nabla_{y} \bar{u}\right|^{q-2} \nabla_{y} \bar{u}\right) \nabla_{y} \varphi\right\}+\int_{\Omega}\left(\underline{u}^{r}-\bar{u}^{r}\right) \varphi \leq 0 .
\end{aligned}
$$

By choosing $\varphi=(\underline{u}-\bar{u})^{+}$as a test function and using the monotonicity of the $p$ and $q$-Laplacians (see [33]), we get that $(\underline{u}-\bar{u})^{+}=0$ and so $\underline{u} \leq \bar{u}$ in $\Omega$.

The relevant results on existence and uniqueness for problem (2.2) are contained in the next theorem.

Theorem 2. Assume that $g \in W^{1, p, q}(\Omega) \cap L^{r+1}(\Omega)$ and $g \geq 0$ on $\partial \Omega$. Then, the problem

$$
\begin{cases}\Delta_{p, q} u=u^{r} & \text { in } \Omega, \\ u=g & \text { on } \partial \Omega,\end{cases}
$$

admits a unique nonnegative solution $u \in W^{1, p, q}(\Omega) \cap L^{r+1}(\Omega)$ in the sense that $u^{r} \varphi \in L^{1}(\Omega)$ and (2.1) is satisfied for arbitrary $\varphi \in W_{0}^{1, p, q}(\Omega)$. In addition, if $g_{m}=\min \{g, m\}, m \in \mathbb{N}$, and $u_{m} \in W^{1, p, q}(\Omega) \cap L^{r+1}(\Omega)$ stands for the solution to (2.3) with $g_{m}$ replacing $g$ then $u_{m} \in L^{\infty}(\Omega)$ and $u_{m} \rightarrow u$ weakly in $W^{1, p, q}(\Omega)$. 
Proof. We first note that uniqueness is a consequence of Lemma 1. We will prove existence of solutions by showing that the functional

$$
J(u)=\int_{\Omega} \frac{1}{p}\left|\nabla_{x} u\right|^{p}+\int_{\Omega} \frac{1}{q}\left|\nabla_{x} u\right|^{q}+\int_{\Omega} \frac{|u|^{r+1}}{r+1} .
$$

achieves its global minimum in $\mathcal{M}:=\left\{u \in W^{1, p, q}(\Omega): u=g\right.$ on $\left.\partial \Omega\right\}$. Since $J$ is sequentially weakly lower semicontinuous -as can be easily shown- and the set $\mathcal{M}$ is weakly closed, the existence of a global minimizer is obtained as soon as we prove that $J$ is coercive (see [31]).

To show that $J$ is coercive, assume on the contrary that there exists a sequence with $\left\|u_{n}\right\| \rightarrow \infty$ and $J\left(u_{n}\right) \leq C$. Then

$$
\int_{\Omega} \frac{t_{n}^{p}}{p}\left|\nabla_{x} v_{n}\right|^{p}+\int_{\Omega} \frac{t_{n}^{q}}{q}\left|\nabla_{y} v_{n}\right|^{q}+\int_{\Omega} t_{n}^{r+1}\left|v_{n}\right|^{r+1} \leq C,
$$

where $t_{n}=\left\|u_{n}\right\|, u_{n}=t_{n} v_{n}$. Passing through a subsequence, $v_{n} \rightarrow v$ weakly in $W^{1, p, q}(\Omega)$ and strongly in $L^{p}(\Omega)$ with $v \in W_{0}^{1, p, q}(\Omega)$. Thus,

$$
\frac{t_{n}^{p-1}}{p} \int_{\Omega}\left|\nabla_{x} v_{n}\right|^{p}+\frac{t_{n}^{q-1}}{q} \int_{\Omega}\left|\nabla_{y} v_{n}\right|^{q}=O(1)
$$

which implies that $v_{n} \rightarrow 0$ strongly in $W^{1, p, q}(\Omega)$. This is not compatible with $\left\|v_{n}\right\|=1$. Therefore, $J$ is coercive and admits a global minimizer.

By Fatou's Lemma $u \in L^{r+1}(\Omega)$ and since $|u| \in \mathcal{M}$ together with $J(|u|)=$ $J(u)$ then we can assume that $u$ is nonnegative. On the other hand, $u$ satisfies

$$
\int_{\Omega}\left|\nabla_{x} u\right|^{p-2} \nabla_{x} u \nabla_{x} \varphi+\left|\nabla_{y} u\right|^{q-2} \nabla_{y} u \nabla_{y} \varphi+\int_{\Omega} u^{r} \varphi=0,
$$

for $\varphi \in C_{0}^{1}(\Omega)$. We claim that test functions $\varphi \in W_{0}^{1, p, q}(\Omega)$ can be inserted in (2.4). To prove this, notice that if $\varphi \in W_{0}^{1, p, q}(\Omega)^{+}$then $\varphi=\lim \varphi_{n}$ in $W_{0}^{1, p, q}(\Omega)$ with $\varphi_{n} \in C_{0}^{1}(\Omega)^{+}$. Since

$$
\int_{\Omega} u^{r} \varphi_{n}=-\left\{\int_{\Omega}\left|\nabla_{x} u\right|^{p-2} \nabla_{x} u \nabla_{x} \varphi_{n}+\left|\nabla_{y} u\right|^{q-2} \nabla_{y} u \nabla_{y} \varphi_{n}\right\},
$$

then Fatou's Lemma implies that $u^{r} \varphi \in L^{1}(\Omega)$. However, this holds true for arbitrary $\varphi \in W_{0}^{1, p, q}(\Omega)$ thanks to the decomposition $\varphi=\varphi^{+}-\varphi^{-}$with $\varphi^{ \pm} \in W_{0}^{1, p, q}(\Omega)^{+}$. Indeed, consider such a $\varphi \in W_{0}^{1, p, q}(\Omega)$ and let $\varphi_{n}$ be a sequence in $C_{0}^{1}(\Omega)$ approximating $\varphi$ in $W_{0}^{1, p, q}(\Omega)$. The equalities

$$
\begin{array}{rl}
\int_{\Omega} u^{r}\left(\varphi_{n}-\varphi_{m}\right)^{ \pm}=-\int_{\Omega}\left|\nabla_{x} u\right|^{p-2} \nabla_{x} & u \nabla_{x}\left(\varphi_{n}-\varphi_{m}\right)^{ \pm} \\
& -\int_{\Omega}\left|\nabla_{y} u\right|^{q-2} \nabla_{y} u \nabla_{y}\left(\varphi_{n}-\varphi_{m}\right)^{ \pm}
\end{array}
$$

hold since the test function $\left(\varphi_{n}-\varphi_{m}\right)^{ \pm} \in L^{\infty}(\Omega)$ and hence it can be inserted in (2.4). Taking into account that

$$
\lim _{m, n \rightarrow \infty} \int_{\Omega} u^{r}\left(\varphi_{n}-\varphi_{m}\right)^{ \pm}=0
$$


we obtain

$$
\lim _{m, n \rightarrow \infty} \int_{\Omega} u^{r}\left|\varphi_{n}-\varphi_{m}\right|=0
$$

This implies that

$$
\begin{aligned}
\int_{\Omega} u^{r} \varphi=\lim \int_{\Omega} u^{r} \varphi_{n} & \\
= & -\left\{\int_{\Omega}\left|\nabla_{x} u\right|^{p-2} \nabla_{x} u \nabla_{x} \varphi+\left|\nabla_{y} u\right|^{q-2} \nabla_{y} u \nabla_{y} \varphi\right\}
\end{aligned}
$$

with an arbitrary $\varphi \in W_{0}^{1, p, q}(\Omega)$, and the claim is proved.

To finish the proof, set $g_{m}=\min \{g, m\}$ and let $u_{m}$ be the solution to (2.3) with $g$ replaced by $g_{m}$. Since $\bar{u}=m$ is a supersolution, by Lemma 1 we have that $u_{m} \leq m$, and thus $u_{m} \in L^{\infty}(\Omega)$. It is also a consequence of Lemma 1 that $u_{m}$ is increasing in $m$ and satisfies $u_{m}(x) \leq u(x)$ a.e. in $\Omega$.

Our next aim will be to obtain estimates for the norms $\left\|u_{m}\right\|$. Taking $\varphi=u_{m}-g_{m}$ as a test function we obtain (a subindex for $\varphi$ is omitted for the sake of clarity)

$$
\begin{gathered}
\int_{\Omega}\left(\left|\nabla_{x} u_{m}\right|^{p-2} \nabla_{x} u_{m}-\left|\nabla_{x} g_{m}\right|^{p-2} \nabla_{x} g_{m}\right) \nabla_{x} \varphi \\
+\int_{\Omega}\left(\left|\nabla_{y} u_{m}\right|^{q-2} \nabla_{y} u_{m}-\left|\nabla_{y} g_{m}\right|^{q-2} \nabla_{y} g_{m}\right) \nabla_{y} \varphi= \\
\quad-\int_{\Omega} u_{m}^{r} \varphi-\int_{\Omega}\left|\nabla_{x} g_{m}\right|^{p-2} \nabla_{x} g_{m} \nabla_{x} \varphi \\
\quad-\int_{\Omega}\left|\nabla_{y} g_{m}\right|^{q-2} \nabla_{y} g_{m} \nabla_{y} \varphi .
\end{gathered}
$$

The right hand side of (2.6) can be estimated by

$$
C(\varepsilon)\left(\left|\nabla_{x} u\right|_{p}^{p}+\left|\nabla_{y} u\right|_{q}^{q}+\left|\nabla_{x} g\right|_{p}^{p}+\left|\nabla_{y} g\right|_{q}^{q}\right)+\varepsilon\left(\left|\nabla_{x} \varphi\right|_{p}^{p}+\left|\nabla_{y} \varphi\right|_{q}^{q}\right),
$$

for a conveniently small $\varepsilon$. In fact, the term involving $u_{m}^{r}$ in (2.6) can be handled, by using (2.5), as follows

$$
\begin{aligned}
\left|\int_{\Omega} u_{m}^{r} \varphi\right| \leq \int_{\Omega} u^{r} \varphi^{+}+ & \int_{\Omega} u^{r} \varphi^{-} \\
& \leq C(\varepsilon)\left(\left|\nabla_{x} u\right|_{p}^{p}+\left|\nabla_{y} u\right|_{q}^{q}\right)+\varepsilon\left(\left|\nabla_{x} \varphi\right|_{p}^{p}+\left|\nabla_{y} \varphi\right|_{q}^{q}\right),
\end{aligned}
$$

where Hölder's and Young's inequalities have been involved elsewhere.

On the other hand, the left hand side in (2.6) can be estimated from below by employing the monotonicity properties of the $p$-Laplacian and $q$ Laplacian operators ([33]). We distinguish several cases. If $q \geq p \geq 2$ such estimate from below reads as follows

$$
C_{p}\left|\nabla_{x} \varphi\right|_{p}^{p}+C_{q}\left|\nabla_{y} \varphi\right|_{q}^{q},
$$

for positive constants $C_{p}, C_{q}$ no depending on $\varphi$. Relation (2.8) combined with (2.7) and a suitable election of $\varepsilon$ imply the boundedness of the sequences $\left|\nabla_{x} \varphi\right|_{p},\left|\nabla_{y} \varphi\right|_{q}, \varphi=g_{m}-u_{m}$. 
Assume now that, say, $1<p<2$. Then, the term involving the $p$ power in the left hand side of (2.6) can be estimated from below by

$$
C_{p}\left\{\frac{\left|\nabla_{x} \varphi\right|_{p}^{p}}{|\Omega|+\left|\nabla_{x} g\right|_{p}^{p}+\left|\nabla_{x} \varphi\right|_{p}^{p}}\right\}^{\frac{2-p}{p}}\left|\nabla_{x} \varphi\right|_{p}^{p},
$$

$C_{p}$ being a positive constant no depending on $\varphi$. Notice that the coefficient of $\left|\nabla_{x} \varphi\right|_{p}^{p}$ in such expression is $O(1)$ and bounded away from zero as $\left|\nabla_{x} \varphi\right|_{p}^{p} \rightarrow$ $\infty$. In addition, the term associated to the power $q$ in the left hand side of $(2.6)$ could be treated exactly in the same way supposed that $1<q<2$ holds. Therefore, the combination of (2.9) and (2.7) and a proper choice of $\varepsilon$ lead again to the boundedness of the sequences $\left|\nabla_{x} \varphi\right|_{p},\left|\nabla_{y} \varphi\right|_{q}, \varphi=u_{m}-g_{m}$. Accordingly, $\left\|u_{m}\right\|$ is bounded.

Thus we have that $u_{m} \rightarrow u^{*}$ weakly in $W^{1, p, q}(\Omega)$ where $u^{*}=\sup u_{m}$. Since $W_{0}^{1, p, q}(\Omega)$ is weakly closed then $u^{*}-g \in W_{0}^{1, p, q}(\Omega)$ and $u^{*}$ is a solution to (2.1) with test functions in $W_{0}^{1, p, q}(\Omega)$. The uniqueness implies that $u^{*}=u$ and the proof is completed.

Remarks 1. (a) The proof of Theorem 2 remains valid if $\Omega$ is unbounded, for instance in the case $\Omega=\mathbb{R}_{+}^{N} \times \mathbb{R}_{+}^{M}$ with $\mathbb{R}_{+}^{N}=\left\{x \in \mathbb{R}^{N}: x_{N}>0\right\}$, $\mathbb{R}_{+}^{M}=\left\{y \in \mathbb{R}^{M}: y_{M}>0\right\}$. In fact, the functional $J(u)$ continues to be coercive in $\mathcal{M}$ (as long as $g \in W^{1, p, q}(\Omega) \cap L^{r+1}(\Omega), g \geq 0$ ), while the general lower semi-continuity result contained in [31] (Theorem 1.6) still allows us to conclude that $J$ exhibits a global and nonnegative minimizer $u \in W^{1, p, q}(\Omega) \cap L^{r+1}(\Omega)$. The same reasoning shows that the weak form (2.4) holds again with test functions in $W_{0}^{1, p, q}(\Omega)$, which is the natural way to obtain uniqueness of solutions in unbounded domains.

(b) The proof of existence can also be obtained using the method of sub and supersolutions as we describe below. One can use the standard iterative technique: if $\underline{u}, \bar{u} \in W^{1, p, q}(\Omega) \cap L^{p^{\prime} r}(\Omega)$ are a sub and a supersolution to (2.2), respectively, such that $0 \leq \underline{u}(x) \leq \bar{u}(x)$ a.e. in $\Omega$, we can construct a sequence by defining $u_{0}=\underline{u}$, and $u=u_{n+1}$, the solution to the problem

$$
\begin{cases}\Delta_{p, q} u=u_{n}^{r} & \text { in } \Omega \\ u=g & \text { on } \partial \Omega .\end{cases}
$$

To show that this problem has a solution one can argue as in Theorem 2 finding a global minimizer of the corresponding energy functional. Lemma 1 then shows that $\underline{u}(x) \leq u_{n}(x) \leq u_{n+1}(x) \leq \bar{u}(x)$, a. e. in $\Omega$ for all $n$. Setting $u(x)=\sup u_{n}(x)$ we find that $u \in L^{p^{\prime} r}(\Omega)$. In addition, the norm of $u_{n}$ can be estimated as in the proof of Theorem 2 and thus, modulus extracting a subsequence, $u_{n} \rightarrow u$, weakly in $W^{1, p, q}(\Omega)$. Hence, $u$ weakly solves the equation in (2.2) while $u-g \in W_{0}^{1, p, q}(\Omega)$. Therefore, $u \in W^{1, p, q}(\Omega) \cap L^{p^{\prime} r}(\Omega)$ is the unique solution to (2.2).

To end this section, we briefly recall some facts on the simpler version of problem (1.1) that occurs when $p=q$,

$$
\begin{cases}\Delta_{p} u(x)=u^{r}(x) & \text { in } Q \\ u(x)=\infty & \text { on } \partial Q,\end{cases}
$$


with $Q \subset \mathbb{R}^{N}$ a smooth bounded domain and $\Delta_{p} u=\operatorname{div}\left(|\nabla u|^{p-2} \nabla u\right)$ the usual $p$-Laplacian, $p>1$. Problem (2.10) will be used later on as an auxiliary tool. Its main properties that are used here are collected in the next statement.

Theorem 3. Let $Q$ be a $C^{2}$ bounded domain of $\mathbb{R}^{N}, p, r$ positive numbers satisfying $p>1$ and

$$
r>p-1 .
$$

Then problem $(2.10)$ has a unique positive weak solution $u \in W_{l o c}^{1, p}(Q)$ such that,

a) $u \in C^{1}(Q) \cap C^{1, \beta}\left(Q^{\prime}\right)$ for every subdomain $Q^{\prime} \subset \bar{Q}^{\prime} \subset Q$ with $\beta$ depending on $Q^{\prime}$. Moreover, the boundary condition is satisfied in the sense that $u(x) \rightarrow$ $\infty$ as $d(x)=\operatorname{dist}(x, \partial Q) \rightarrow 0$.

b) The solution u satisfies:

$$
\lim _{d(x) \rightarrow 0} \frac{u(x)}{A d(x)^{-\alpha}}=1,
$$

where $d(x)=\operatorname{dist}(x, \partial Q)$ and $\alpha$ and $A$ depend explicitly on $p$ and $r$,

$$
\alpha=\frac{p}{r-p+1}, \quad A^{r-p+1}=(p-1) \alpha^{p-1}(\alpha+1) .
$$

Remark 2. A proof of Theorem 3 is essentially contained in [8] where it is shown that all possible solutions exhibit the asymptotic profile (2.11). With this asymptotic profile at hand, uniqueness is readily obtained by using the direct argument in [15] instead of the approach in [8]. Moreover, the restrictions on the geometry of $\partial Q$ required in 8 . for existence of solutions to $(2.10)$ can be omitted by employing the "up to the boundary" $C^{1, \beta}$ estimates in [21]. On the other hand, more general nonlinearities are studied in [25]. However, the uniqueness of solutions to (2.10) with more general nonlinearities is only obtained in rather special domains. Finally, the regularity assertion in a) can be considerably improved since it can be shown that the gradient $\nabla u$ of $u$ also blows up in modulus when $x$ approaches $\partial Q$ (cf. [6] for the semilinear case $p=2$ ). This implies that the equation becomes non degenerate in the elliptic sense in a neighborhood of $\partial Q$. Then, the standard theory of quasilinear elliptic equations (cf. [19]) permits to conclude that $u$ upgrades regularity up to $C^{2}$ in such neighborhood and in particular $u$ is of class $C^{1+\beta}$ in the whole $Q$ for a certain $\beta \in(0,1)$.

\section{The CASE of PRoduct Domains}

This section is devoted to the analysis of the main features of problem (1.1) in "box" domains $\Omega=\Omega_{1} \times \Omega_{2}$.

Theorem 4. Let $\Omega=\Omega_{1} \times \Omega_{2}$, where $\Omega_{1} \subset \mathbb{R}^{N}$ and $\Omega_{2} \subset \mathbb{R}^{M}$ are bounded $C^{2}$ domains, $q \geq p>1$ and $r>q-1$. Then, problem (1.1) admits a minimal solution $u \in W_{\text {loc }}^{1, p, q}(\Omega) \cap L_{\text {loc }}^{\infty}(\Omega)$. Moreover, for each $g \in W^{1, p, q}(\Omega) \cap L^{r+1}(\Omega)$ we have that

$$
u_{g} \leq u
$$


a.e. in $\Omega$ where $u_{g} \in W^{1, p, q}(\Omega)$ stands for the solution to the finite boundary problem (2.3).

Remark 3. It will be proved later in Theorem 9 that $r>\max \{p-1, q-1\}$ also becomes a necessary condition for the existence of solutions to (1.1) on a general bounded domain $\Omega \subset \mathbb{R}^{N} \times \mathbb{R}^{M}$.

Proof. First, we construct a smooth supersolution $\bar{u}$ to the equation $\Delta_{p, q} u=$ $u^{r}$ in $\Omega$ which blows up on $\partial \Omega$. To this end, let $U \in C^{1, \beta}\left(\Omega_{1}\right)$ be the solution to problem (2.10) with $Q=\Omega_{1}$ while $V \in C^{1, \beta}\left(\Omega_{2}\right)$ will designate the solution to

$$
\begin{cases}\Delta_{q} u(x)=u^{r}(x) & \text { in } \Omega_{2} \\ u(x)=\infty & \text { on } \partial \Omega_{2} .\end{cases}
$$

Then, we have that

$$
\bar{u}=t U+s V,
$$

$t, s \geq 1$, defines a supersolution provided either $t$ or $s$ are conveniently large. In fact, take $\varphi \in C_{0}^{1}(\Omega)^{+}$. Then, for every $y \in \Omega_{2}$,

$$
\int_{\Omega_{1}} t^{p-1}|\nabla U|^{p-2} \nabla U \nabla_{x} \varphi(\cdot, y)=-\int_{\Omega_{1}} t^{p-1} U^{r} \varphi(\cdot, y),
$$

and, similarly, for each $x \in \Omega_{1}$ it holds

$$
\int_{\Omega_{2}} s^{q-1}|\nabla V|^{q-2} \nabla V \nabla_{y} \varphi(x, \cdot)=-\int_{\Omega_{2}} s^{q-1} V^{r} \varphi(x, \cdot) .
$$

Integrating the first equality in $\Omega_{2}$, the second one in $\Omega_{1}$ and adding the results we get

$$
\begin{aligned}
\int_{\Omega}\left|\nabla_{x} \bar{u}\right|^{p-2} \nabla_{x} \bar{u} \nabla_{x} \varphi & +\left|\nabla_{y} \bar{u}\right|^{q-2} \nabla_{y} \bar{u} \nabla_{y} \varphi \\
& =-\int_{\Omega}\left(t^{p-1} U^{r}+s^{q-1} V^{r}\right) \varphi \geq-\int_{\Omega}(t U+s V)^{r} \varphi .
\end{aligned}
$$

Here we have used the fact that the inequality $t^{p-1} x^{r}+s^{q-1} y^{r} \leq(t x+s y)^{r}$ holds for all $x, y>0$ if $t, s \geq 1$ and it is assumed that either $t$ or $s$ are large enough (recall that $r>q-1 \geq p-1$ ). Thus, $\bar{u}$ defines a supersolution.

We next show that any finite solution to the equation is bounded by $\bar{u}$. In fact, let $u_{g} \in W^{1, p, q}(\Omega) \cap L^{r+1}(\Omega)$ be the solution to (2.3). We first assume that $g \in L^{\infty}(\Omega)$. In this case,

$$
u_{g} \leq u_{\|g\|_{\infty}} \leq\|g\|_{\infty}
$$

where $u_{\|g\|_{\infty}}$ stands for the solution to (2.3) with $g$ replaced by $\|g\|_{\infty}$. Lemma 1 implies $u_{g} \leq \bar{u}$. Finally, to obtain this inequality for a general $g$, we use the approximation result in Theorem 2 . Thus, setting $g_{n}=\min \{n, g\}$ we first obtain $u_{g_{n}} \leq \bar{u}$ in $\Omega$ for each $n$ and then, by taking supremum, $u_{g} \leq \bar{u}$.

Next, we construct the minimal solution of problem (1.1). To this end, if $u=u_{m} \in W^{1, p, q}(\Omega) \cap L^{\infty}(\Omega)$ is the solution to

$$
\begin{cases}\Delta_{p, q} u(x, y)=u^{r}(x, y) & \text { in } \Omega, \\ u(x, y)=m & \text { on } \partial \Omega\end{cases}
$$


then $\left\{u_{m}\right\}$ is an increasing sequence, such that

$$
u_{m}(x, y) \leq \bar{u}(x, y) \quad(x, y) \in \Omega .
$$

Thus

$$
u(x, y)=: \lim _{m \rightarrow \infty} u_{m}(x, y)=\sup _{m} u_{m}(x, y)<\infty \quad(x, y) \in \Omega,
$$

is a well defined function in $L_{l o c}^{\infty}(\Omega)$.

Let us introduce now the sets $A_{k}=\{\bar{u}<k\}(k \in \mathbb{N})$. Since $\cup A_{k}=\Omega$, any subdomain $\Omega^{\prime} \subset \overline{\Omega^{\prime}} \subset \Omega$, such that $\overline{\Omega^{\prime}}$ is compact lies in a certain $A_{k}$ for $k$ large enough. Setting in addition $A_{k}^{m}=\left\{u_{m}<k\right\}$, we find that

$$
A_{k} \subset A_{k}^{m+1} \subset A_{k}^{m},
$$

for every $m$.

Using $\varphi=\left(k-u_{m}\right)^{+}, m>k$, as a test function in the weak form of the equation verified by $u_{m}$ we obtain

$$
\int_{A_{k}^{m}}\left|\nabla_{x} u_{m}\right|^{p}+\left|\nabla_{y} u_{m}\right|^{q}=\int_{A_{k}^{m}} u_{m}^{r} \varphi
$$

This implies that

$$
\int_{\Omega^{\prime}}\left|\nabla_{x} u_{m}\right|^{p}+\left|\nabla_{y} u_{m}\right|^{q} \leq k^{r+1}|\Omega|
$$

and hence $\left\|u_{m}\right\|$ is bounded in $\Omega^{\prime}$ which implies that $u$ is the weak limit of $u_{m}$ in $W^{1, p, q}\left(\Omega^{\prime}\right)$. Since $\Omega^{\prime}$ is arbitrary we conclude that $u \in W_{l o c}^{1, p, q}(\Omega)$ and passing to the limit in the weak formulation of (3.2) we see that $u$ defines a weak solution to $\Delta_{p, q} u=u^{r}$ in $\Omega$. To check that the boundary condition is satisfied in the weak sense we observe that for fixed $k>0$,

$$
(k-u)^{+}=\lim _{m \rightarrow \infty}\left(k-u_{m}\right)^{+},
$$

pointwise in $\Omega$, with $\left(k-u_{m}\right)^{+} \in W_{0}^{1, p, q}(\Omega)$ for every $m>k$. Taking $\varphi_{m}=\left(k-u_{m}\right)^{+}$as a test function we obtain

$$
\int_{\Omega}\left|\nabla_{x} \varphi_{m}\right|^{p}+\left|\nabla_{y} \varphi_{m}\right|^{q}=\int_{\Omega} u_{m}^{r} \varphi_{m} \leq k^{r+1}|\Omega|
$$

and since $W_{0}^{1, p, q}(\Omega)$ is weakly closed this means that $(k-u)^{+} \in W_{0}^{1, p, q}(\Omega)$, as desired.

Now, let $u_{g}$ be the solution to (2.3) with $g \in W^{1, p, q}(\Omega) \cap L^{r+1}(\Omega)$. According to Theorem 2 we have $u_{g}=\lim u_{g_{n}}$ with $g_{n} \in L^{\infty}(\Omega)$. Since $u_{g_{n}} \leq u_{\left\|g_{n}\right\|_{\infty}} \leq u$ we get $\lim _{n} u_{g_{n}} \leq u$.

To conclude the proof, we need to show that the solution $u$ constructed above is the minimal solution. To this end, let $v$ be an arbitrary solution, and notice that by Lemma $1 u_{m} \leq v$ in $\Omega$. Letting $m \rightarrow \infty$ we arrive at $u \leq v$, as we wanted to prove.

Remark 4. Theorem 4 remains true if one (or both) of the domains $\Omega_{1}, \Omega_{2}$ is unbounded. The main requirement is the solvability of problem (2.10) in $Q=\Omega_{1}$ in case $\Omega_{1}$ is unbounded (respectively in $Q=\Omega_{2}$ with $q$ replacing $p$, in case $\Omega_{2}$ is unbounded). For instance, if $\Omega=\mathbb{R}_{+}^{N} \times \mathbb{R}_{+}^{M}$ then the proof of Theorem 4 works without changes. In fact, solutions $u_{m}$ are constructed by 
using Remark 1 and Theorem 2 while to obtain the supersolution $\bar{u}$ one uses the solutions $U=U(x)$ and $V=V(y)$ to $\Delta_{p} u=u^{p}$ in $\mathbb{R}_{+}^{N}, u_{\mid x_{N}=0}=\infty$ and $\Delta_{q} u=u^{p}$ in $\mathbb{R}_{+}^{M}, u_{\left.\right|_{M}=0}=\infty$, respectively. These functions are explicit. In fact, it can be checked that $U(x)=A x_{N}^{-\alpha}, \alpha, A$ as in Theorem 3, while $V(y)=B y_{M}^{-\beta}$ with $\beta=q / r-q+1$ and $B^{r-q+1}=(q-1) \beta^{q-1}(\beta+1)$.

Next, let us consider an issue which is interesting in its own right. Namely, the study of the asymptotic profiles of solutions to (1.1) near the boundary. For this purpose, and also by its intrinsic interest, we state first a uniqueness result for problem (1.1) which holds in a large class of domains of the form $\Omega_{1} \times \Omega_{2}$. Its proof is inspired by an idea contained in $[20$.

Theorem 5. Let $\Omega_{1} \subset \mathbb{R}^{N}, \Omega_{2} \subset \mathbb{R}^{M}$ be bounded starshaped domains with respect to the points $\xi_{1} \in \Omega_{1}, \xi_{2} \in \Omega_{2}$, and $r>q-1 \geq p-1$. Then, problem (1.1) possesses a unique positive solution in $\Omega=\Omega_{1} \times \Omega_{2}$.

Proof. No generality is lost if it is assumed that $\xi_{1}=0 \in \mathbb{R}^{N}, \xi_{2}=0 \in \mathbb{R}^{M}$. Suppose $u, v \in W_{l o c}^{1, p, q}(\Omega) \cap L_{l o c}^{\infty}(\Omega)$ are positive solutions to (1.1).

For positive $\lambda, \mu$ set $\Omega_{1, \lambda}=\left\{\lambda x: x \in \Omega_{1}\right\}, \Omega_{2, \mu}=\left\{\mu y: y \in \Omega_{2}\right\}$ and take

$$
\Omega_{\lambda, \mu}=\Omega_{1, \lambda} \times \Omega_{2, \mu} .
$$

Define

$$
\bar{u}(\bar{x}, \bar{y})=\lambda^{-\frac{p}{r-p+1}} u\left(\frac{\bar{x}}{\lambda}, \frac{\bar{y}}{\mu}\right)
$$

with $(\bar{x}, \bar{y}) \in \Omega_{\lambda, \mu}$ and where

$$
\mu=\lambda^{\frac{p}{q} \frac{r-q+1}{r-p+1}} .
$$

Then, we find that $\bar{u}$ solves problem (1.1) in $\Omega_{\lambda, \mu}$. If $0<\lambda<1$ then $\Omega_{\lambda, \mu} \subset \bar{\Omega}_{\lambda, \mu} \subset \Omega$ while $v=v(x, y)$ becomes a finite solution to $\Delta_{p, q} u=u^{r}$ in $\Omega_{\lambda, \mu}$. Hence, by Lemma 1

$$
v(x, y) \leq \bar{u}(x, y) \quad(x, y) \in \Omega_{\lambda, \mu} .
$$

Since $\Omega_{\lambda, \mu} \rightarrow \Omega$ as $\lambda \rightarrow 1$ this implies that $v(x, y) \leq u(x, y)$ a.e. in $\Omega$. The reversed inequality is shown in the same way and then uniqueness is proved.

Remarks 5. (a) A reference case where the hypotheses of Theorem 5 hold is certainly the product of two balls in $\mathbb{R}^{N}$ and $\mathbb{R}^{M}$ respectively, $B_{1}\left(\xi_{1}, R_{1}\right) \times$ $B_{2}\left(\xi_{2}, R_{2}\right)$. The unique solution $u$ to (1.1) in this domain takes the form

$$
u(x, y)=v\left(r_{1}, r_{2}\right)
$$

where $r_{1}=\left|x-\xi_{1}\right|, r_{2}=\left|y-\xi_{2}\right|$. Notice also that $u(x, y)=v(|x|,|y|)$ gives the unique solution to (1.1) in the shifted ball $B_{1}\left(0, R_{1}\right) \times B_{2}\left(0, R_{2}\right)$.

(b) A second reference situation where uniqueness is attained is $\Omega=$ $\mathbb{R}_{+}^{N} \times \mathbb{R}_{+}^{M}$ (see Remark 4). Thus in this domain the solution to problem (1.1) is given by

$$
u(x, y)=v\left(x_{N}, y_{M}\right),
$$

where $v$ stands for the positive solution to (1.1) in $\Omega=\mathbb{R}_{+}^{2}$. 
The behavior of the minimal solution $u(x, y)$ to problem (1.1) when $(x, y)$ approaches $\partial \Omega$ is described in our next result. We remark that we are not considering the case where $(x, y)$ converges to a "corner point" $\left(x_{0}, y_{0}\right) \in$ $\partial \Omega_{1} \times \partial \Omega_{2}$ on the boundary (for this case see the analysis of the particular cases $\Omega=\mathbb{R}_{+}^{N} \times \mathbb{R}_{+}^{M}, \Omega=\mathbb{R}_{+}^{2}$ at the end of the section).

Theorem 6. Assume $\Omega_{1} \subset \mathbb{R}^{N}, \Omega_{2} \subset \mathbb{R}^{M}$ are bounded smooth domains, $r>q-1 \geq p-1$. If $u(x, y)$ is the minimal solution to (1.1) in $\Omega=\Omega_{1} \times \Omega_{2}$ and $\left(x_{0}, y_{0}\right) \in\left(\partial \Omega_{1} \times \Omega_{2}\right) \cup\left(\Omega_{1} \times \partial \Omega_{2}\right)$ then

$$
\lim _{(x, y) \rightarrow\left(x_{0}, y_{0}\right)} d(x, y)^{\gamma} u(x, y)=B
$$

where $d(x, y)=\operatorname{dist}((x, y), \partial \Omega)$ and

$$
\gamma=\frac{p}{r-p+1}, \quad B^{r-p+1}=(p-1) \gamma^{p-1}(\gamma+1),
$$

if $\left(x_{0}, y_{0}\right)$ is a "vertical" point, i.e. $\left(x_{0}, y_{0}\right) \in \partial \Omega_{1} \times \Omega_{2}$, while

$$
\gamma=\frac{q}{r-q+1}, \quad B^{r-q+1}=(q-1) \gamma^{q-1}(\gamma+1),
$$

provided $\left(x_{0}, y_{0}\right)$ is a "horizontal" point $\left(x_{0}, y_{0}\right) \in \Omega_{1} \times \partial \Omega_{2}$.

Remark 6. For future use we are setting $\alpha, A_{p}$ for the values of $\gamma$ and $B$ in the vertical case and $\beta, A_{q}$ for the corresponding values in the horizontal case.

Proof. We analyze the case $\left(x_{0}, y_{0}\right) \in \partial \Omega_{1} \times \Omega_{2}$, the other one being handled similarly. Thus, let $U_{m} \in W^{1, p}\left(\Omega_{1}\right) \cap C^{1, \beta}\left(\bar{\Omega}_{1}\right)$ be the unique positive solution to

$$
\left\{\begin{array}{c}
\Delta_{p} u(x)=u^{r}(x) \quad \text { in } \Omega_{1} \\
u(x)=m \quad \text { on } \partial \Omega_{1} .
\end{array}\right.
$$

Since $\underline{u}(x, y)=U_{m}(x)$ defines a subsolution to (3.2) then

$$
U_{m}(x) \leq u_{m}(x, y) \leq u(x, y) \quad(x, y) \in \Omega,
$$

where $u$ stands for the minimal solution to (1.1). This implies that $U(x) \leq$ $u(x, y)$ in $\Omega$, where $U(x)$ is the solution to (2.10) in $Q=\Omega_{1}$. Since

$$
d(x, y)=d_{1}(x)=: \operatorname{dist}\left(x, \partial \Omega_{1}\right)
$$

as $(x, y) \rightarrow\left(x_{0}, y_{0}\right)$, then

$$
\varliminf_{(x, y) \rightarrow\left(x_{0}, y_{0}\right)} d(x, y)^{\alpha} u(x, y) \geq \lim _{x \rightarrow x_{0}} d_{1}(x)^{\alpha} U(x)=A_{p},
$$

where $\alpha$ and $A_{p}$ stand for the exponent and constant in the vertical case of the statement of Theorem 3, respectively.

To obtain the complementary estimate we use again the supersolution $\bar{u}(x, y)=t U(x)+s V(y)$ introduced in the proof of Theorem 4 with the choices $t=1+\varepsilon, \varepsilon>0$ and $s \geq 1$ sufficiently large. It was shown there that

$$
u(x, y) \leq \bar{u}(x, y) .
$$

Therefore,

$$
\varlimsup_{(x, y) \rightarrow\left(x_{0}, y_{0}\right)} d(x, y)^{\alpha} u(x, y) \leq(1+\varepsilon) A
$$

and estimate (3.3) follows by doing $\varepsilon \rightarrow 0$. 
A consequence of Theorem 6 is the following result.

Corollary 7. Under the conditions of Theorem 6 assume in addition that $\Omega_{1}, \Omega_{2}$ are starshaped (for example, balls, half spaces, convex sets). Then, the unique solution $u$ to (1.1) in $\Omega_{1} \times \Omega_{2}$ satisfies the asymptotic estimates described in (3.3).

Remarks 7. (a) Using the notation of Remark 5, when $\Omega=B_{1}\left(\xi_{1}, R_{1}\right) \times$ $B_{2}\left(\xi_{1}, R_{1}\right)$ and, say $\left(x_{0}, y_{0}\right) \in \partial B_{1}\left(\xi_{1}, R_{1}\right) \times B_{2}\left(\xi_{1}, R_{1}\right)$, then it follows from (3.3) that

$$
\lim \left(R_{1}-\left|x_{n}-\xi_{1}\right|\right)^{\gamma} v\left(\left|x_{n}-\xi_{1}\right|,\left|y_{n}-\xi_{1}\right|\right)=B,
$$

when $\left(x_{n}, y_{n}\right) \in B_{1}\left(\xi_{1}, R_{1}\right) \times B_{2}\left(\xi_{1}, R_{1}\right)$ converges to $\left(x_{0}, y_{0}\right)$.

On the other hand, if $v(x, y)$ stands for the positive solution to (1.1) in $\mathbb{R}_{+}^{2}$ then

$$
\lim _{(x, y) \rightarrow\left(0, y_{0}\right)} x^{-\alpha} u(x, y)=A_{p}, \quad \lim _{(x, y) \rightarrow\left(x_{0}, 0\right)} y^{-\beta} u(x, y)=A_{q},
$$

with $y_{0}>0$ and $x_{0}>0$; the exponents $\alpha, \beta$ and coefficients $A_{p}, A_{q}$ are given in Theorem 6 .

(b) The proof of Theorem $[6$ shows that the lower estimate

$$
\varliminf_{(x, y) \rightarrow\left(x_{0}, y_{0}\right)} d(x, y)^{\gamma} u(x, y) \geq B,
$$

holds true for an arbitrary (not only for the minimal) positive solution $u \in$ $W_{l o c}^{1, p, q}(\Omega) \cap L_{l o c}^{\infty}(\Omega)$ to (1.1).

(c) By using the previous characterization of the asymptotic profile of the solution to (1.1) in domains $B_{1} \times B_{2}-B_{1}, B_{2}$ balls-, the autonomous character of the equation in (1.1) and a sweeping argument (as in [7]), it can also be shown that an arbitrary solution $u$ to (1.1) in a domain $\Omega_{1} \times \Omega_{2}$ satisfies

$$
\varlimsup_{(x, y) \rightarrow\left(x_{0}, y_{0}\right)} d(x, y)^{\gamma} u(x, y) \leq B,
$$

provided that $(x, y)$ approaches, say $\left(x_{0}, y_{0}\right) \in \partial \Omega_{1} \times \Omega_{2}$, in a "cone" $\langle x-$ $\left.x_{0},-\nu_{1}\left(x_{0}\right)\right\rangle \geq \theta\left|x-x_{0}\right|$, with $\nu_{1}\left(x_{0}\right)$ the outward unit normal to $\partial \Omega_{1}$ at $x_{0}$ and $\theta \in(0,1)$ a constant.

We are now in a position to prove that the upper estimate (3.5) holds true for arbitrary solutions to problem (1.1) without restricting the conditions on the convergence to a point on $\partial \Omega$.

Theorem 8. Suppose $\Omega_{1} \subset \mathbb{R}^{N}, \Omega_{2} \subset \mathbb{R}^{M}$ are smooth bounded domains, $1<p \leq q, r>q-1$, and let $z_{0}=\left(x_{0}, y_{0}\right)$ be a boundary point of $\Omega_{1} \times \Omega_{2}$ not lying on the "corner" $\partial \Omega_{1} \times \partial \Omega_{2}$. Then, any positive solution $u \in$ $W_{l o c}^{1, p, q}(\Omega) \cap L_{l o c}^{\infty}(\Omega)$ to (1.1) satisfies the asymptotic estimate (3.3).

Proof. Assume $z_{0}=\left(x_{0}, y_{0}\right) \in \partial \Omega_{1} \times \Omega_{2}$ and $z_{n}=\left(x_{n}, y_{n}\right) \rightarrow z_{0}$ with $z_{n} \in \Omega$. Choose $\bar{z}_{n}=\left(\bar{x}_{n}, y_{n}\right), \bar{x}_{n} \in \partial \Omega_{1}$, such that the distance $d\left(x_{n}, y_{n}\right)$ form $z_{n}$ to $\partial \Omega$ is given by

$$
d\left(z_{n}\right)=\left|x_{n}-\bar{x}_{n}\right|
$$


for all $n$. The smoothness assumption on $\Omega_{1}$ allows us to get the existence of a positive radius $R$ such that:

1) $d\left(z_{n}\right)<R$ for large $n$,

2) setting $\xi_{n}=\bar{x}_{n}-R \nu\left(\bar{x}_{n}\right)$, $\nu$ the outward unit normal on $\partial \Omega_{1}$ then $B_{1}\left(\xi_{n}, R\right) \subset \Omega_{1}$ while $\partial B_{1}\left(\xi_{n}, R\right) \cap \partial \Omega_{1}=\left\{\bar{x}_{n}\right\}$,

3) $B_{2}\left(y_{n}, R\right) \subset \overline{B_{2}\left(y_{n}, R\right)} \subset \Omega_{2}$ for all $n$.

Letting $v_{n}(x, y)=v\left(\left|x-\xi_{n}\right|,\left|y-y_{n}\right|\right)$ the solution to (1.1) in $B_{1}\left(\xi_{n}, R\right) \times$ $B_{2}\left(y_{n}, R\right)$ (Remarks 5 and 7 ) we get, after sweeping, that

$$
u(x, y) \leq v_{n}(x, y)
$$

for $(x, y) \in B_{1}\left(\xi_{n}, R\right) \times B_{2}\left(y_{n}, R\right)$ and all $n$. Thus,

$$
d\left(z_{n}\right)^{\gamma} u\left(x_{n}, y_{n}\right) \leq d\left(z_{n}\right)^{\gamma} v\left(\left|x_{n}-\xi_{n}\right|, 0\right)=d\left(z_{n}\right)^{\gamma} v\left(R-d\left(z_{n}\right), 0\right) .
$$

Taking limits we arrive at

$$
\varlimsup
$$

which is (3.5). As the complementary estimate always holds (Remark 7) the proof is finished.

We conclude our analysis of asymptotic profiles of solutions to (1.1) in box-type domains by giving an insight of the possible behavior near "corner points" on $\partial \Omega$ (i. e. points on $\partial \Omega_{1} \times \partial \Omega_{2}$ ). For this purpose we are dealing with the special case of $\Omega=\mathbb{R}_{+}^{N} \times \mathbb{R}_{+}^{M}$. In view of Remarks 5 (b) it suffices with analyzing directly the case $\Omega=\mathbb{R}_{+}^{2}$.

Accordingly, let us observe that if $u(x, y)$ is a positive solution to

$$
\left\{\begin{array}{cl}
\left(\left|u_{x}\right|^{p-2} u_{x}\right)_{x}(x, y)+\left(\left|u_{y}\right|^{q-2} u_{y}\right)_{y}(x, y)=u^{r}(x, y) & (x, y) \in \mathbb{R}_{+}^{2} \\
u(x, y)=\infty & (x, y) \in \partial \mathbb{R}_{+}^{2},
\end{array}\right.
$$

then, for every positive $\lambda>0$,

$$
u_{\lambda}(x, y)=: \lambda^{\alpha} u\left(\lambda x, \lambda^{\alpha / \beta} y\right),
$$

also defines a positive solution to (3.6), where the exponents $\alpha$ and $\beta$ are given by $\alpha=p / r-p+1, \beta=q / r-q+1$.

It follows by uniqueness that

$$
u(x, y)=\lambda^{\alpha} u\left(\lambda x, \lambda^{\alpha / \beta} y\right),
$$

for all $(x, y) \in \mathbb{R}_{+}^{2}$ and positive $\lambda$. Setting in particular $\lambda=1 / x$ we conclude that the solution has the self-similar form,

$$
u(x, y)=x^{-\alpha} f\left(x^{-\alpha / \beta} y\right),
$$

where $f \in C(0, \infty)$ is positive and absolutely continuous in every compact interval $I \subset \mathbb{R}^{+}$.

It follows from Corollary 7 (see Remarks 7 ) that

$$
\lim _{t \rightarrow \infty} f(t)=A_{p}, \quad \text { while } \quad \lim _{t \rightarrow 0+} t^{\beta} f(t)=A_{q} .
$$


Thus, the behavior of $u(x, y)$ near the corner point $(0,0)$ is summarized as follows,

$$
u(x, y) \sim \begin{cases}A_{p} x^{-\alpha} & y \gg x^{\alpha / \beta} \\ f\left(t^{*}\right) x^{-\alpha} & y \sim t^{*} x^{\alpha / \beta} \\ A_{q} y^{-\beta} & y \ll x^{\alpha / \beta},\end{cases}
$$

where $t^{*}>0$ and $x \rightarrow 0+$. Here we use the following notation: for functions $a(s)$ and $b(s)$ defined near $s=0$ by $a(s) \gg b(s)$ it is understood -as usualthat $a(s) / b(s) \rightarrow \infty$ as $s \rightarrow 0$ while $a(s) \sim b(s)$ means $a(s) / b(s) \rightarrow 1$ as $s \rightarrow 0$. Accordingly, $y \gg x^{\alpha / \beta}$ means that $y$ is any function of $x$ near zero so that the asymptotic relation described above holds as $x \rightarrow 0+$.

\section{Problem (1.1) in general domains}

In the present section we deal with the singular problem (1.1) in a general smooth bounded domain $\Omega \subset \mathbb{R}^{N} \times \mathbb{R}^{M}$. Our main result is the following:

Theorem 9. Assume $\Omega \subset \mathbb{R}^{N} \times \mathbb{R}^{M}$ is bounded and smooth, and $q \geq$ $p>1$. A necessary and sufficient condition for the existence of a solution $u \in W_{l o c}^{1, p, q}(\Omega) \cap L_{l o c}^{\infty}(\Omega)$ to the problem

$$
\begin{cases}\Delta_{p, q} u(x, y)=u^{r}(x, y) & \text { in } \Omega, \\ u(x, y)=+\infty & \text { on } \partial \Omega,\end{cases}
$$

is

$$
r>\max \{p-1, q-1\} .
$$

Provided that such condition holds then (1.1) possesses a minimal solution $u \in W_{l o c}^{1, p, q}(\Omega) \cap L_{l o c}^{\infty}(\Omega)$ such that

$$
v(x, y) \leq u(x, y) \quad(x . y) \in \Omega,
$$

for every "finite" solution $v \in W^{1, p, q}(\Omega)$ to the equation $\Delta_{p, q} v=v^{r}$ in $\Omega$.

Proof. To show the existence of a solution consider as before the (increasing) sequence of functions $u_{m} \in W^{1, p, q}(\Omega), m \in \mathbb{N}, u=u_{m}$ the solution to

$$
\left\{\begin{array}{cl}
\Delta_{p, q} u(x, y)=u^{r}(x, y) & \text { in } \Omega \\
u(x, y)=m & \text { on } \partial \Omega .
\end{array}\right.
$$

Then

$$
u(x, y)=\lim _{m \rightarrow \infty} u_{m}(x, y)=\sup _{m} u_{m}(x, y)
$$

is finite at every $(x, y) \in \Omega$ and $u$ defines a function in $L_{l o c}^{\infty}(\Omega)$. Indeed, this immediately follows from the fact that for every cube-type domain $Q=$ $\Omega_{1} \times \Omega_{2}, Q$ as in Theorem $4, Q \subset \bar{Q} \subset \Omega$, we have

$$
u_{m}(x, y) \leq u_{Q}(x, y) \quad(x, y) \in Q,
$$

$u_{Q} \in W_{l o c}^{1, p, q}(Q)$ being the minimal solution to (1.1) in $Q$.

It can be shown exactly as in the proof of Theorem 4 that the function $u$ defined above belongs to $W_{l o c}^{1, p, q}(\Omega)$ and is the minimal solution to (1.1). 
Let us show now the sufficiency of condition (4.1). Suppose that a solution $u \in W_{l o c}^{1, p, q}(\Omega)$ to (1.1) exists and consider bounded smooth domains $\Omega_{1} \subset$ $\mathbb{R}^{N}, \Omega_{2} \subset \mathbb{R}^{M}$ such that $\Omega \subset \Omega_{1} \times \Omega_{2}$. For $m \in \mathbb{N}$, set $u=U_{m} \in W^{1, p}\left(\Omega_{1}\right) \cap$ $C^{1, \beta}\left(\bar{\Omega}_{1}\right)$ the solution to

$$
\left\{\begin{array}{cl}
\Delta_{p} u(x)=u^{r}(x) & \text { in } \Omega_{1} \\
u(x)=m & \text { on } \partial \Omega_{1},
\end{array}\right.
$$

while $v=V_{n} \in W^{1, q}\left(\Omega_{2}\right) \cap C^{1, \beta}\left(\bar{\Omega}_{2}\right)(n \in \mathbb{N})$ stands for the solution to the problem

$$
\left\{\begin{array}{cl}
\Delta_{q} v(y)=v^{r}(y) & \text { in } \Omega_{2} \\
u(y)=n & \text { on } \partial \Omega_{2} .
\end{array}\right.
$$

If $u_{m} \in W^{1, p, q}(\Omega)$ is the solution to $\Delta_{p, q} u=u^{r}$ defined in the first part of the proof then one finds that for every $m \in \mathbb{N}$

$$
U_{m}(x) \leq u_{m}(x, y) \leq u(x, y) \quad(x, y) \in \Omega,
$$

and similarly

$$
V_{n}(y) \leq u_{n}(x, y) \leq u(x, y) \quad(x, y) \in \Omega,
$$

$n \in \mathbb{N}$ arbitrary.

We are next showing that, the condition $r \leq p-1$ implies that

$$
\lim _{m \rightarrow \infty} U_{m}=\infty
$$

uniformly in $\Omega_{1}$. In fact, setting $w_{m}=U_{m} / m$ we obtain the problem

$$
\begin{cases}\Delta_{p} w_{m}=m^{r-p+1} w_{m}^{r} & \text { in } \Omega_{1} \\ w_{m}=1 & \text { on } \partial \Omega_{1} .\end{cases}
$$

Since $m^{r-p+1} w_{m}^{r} \leq w_{m}^{r}$, we obtain

$$
w_{m}(x) \geq w(x) \quad x \in \Omega_{1},
$$

where $w \in W^{1, p}(\Omega) \cap C^{1, \alpha}\left(\bar{\Omega}_{1}\right), w>0$ in $\Omega_{1}$, is the solution to

$$
\left\{\begin{array}{cl}
\Delta_{p} w(x)=w^{r}(x) & \text { in } \Omega_{1} \\
w(x)=1 & \text { on } \partial \Omega_{1} .
\end{array}\right.
$$

In particular $U_{m}(x) \geq m w(x)$ for $x \in \Omega_{1}$ which implies that $U_{m} \rightarrow \infty$ uniformly in $\Omega_{1}$.

The same argument shows that $V_{n} \rightarrow \infty$ uniformly in $\Omega_{2}$ provided $r \leq$ $q-1$. This means that existence of solutions to (1.1) is not compatible with the failure of condition (4.1).

As for the uniqueness issue let us make a few remarks. The main feature is that the proof of uniqueness of solutions to (1.1) in Theorem 5 remains valid without changes provided the image $\Omega_{\lambda, \lambda^{\alpha / \beta}}$ of the reference domain $\Omega$, under the scaling transformation

$$
(x, y) \rightarrow\left(x^{\prime}, y^{\prime}\right), \quad x^{\prime}=\lambda\left(x-x_{0}\right)+x_{0}, \quad y^{\prime}=\lambda^{\alpha / \beta}\left(y-y_{0}\right)+y_{0},
$$

lies again in $\Omega$ for all $0<\lambda<1$ and a certain point $\left(x_{0}, y_{0}\right) \in \Omega$. 
To have an insight of the meaning of such invariance property let us present some examples. For a domain $\Omega \subset \mathbb{R}^{N} \times \mathbb{R}^{M}$ let us designate by $\Omega_{1} \subset \mathbb{R}^{N}, \Omega_{2} \subset \mathbb{R}^{M}$ its projections onto $\mathbb{R}^{N}$ and $\mathbb{R}^{M}$, respectively. For $\xi \in \Omega_{1}, \eta \in \Omega_{2}$ define the corresponding sections as $\Omega_{2, \xi}=\{y:(\xi, y) \in \Omega\}$, $\Omega_{1, \eta}=\{x:(x, \eta) \in \Omega\}$. A sufficient condition in order that a convex domain $\Omega$ exhibits such property with respect to $\left(x_{0}, y_{0}\right)$ is that $x_{0} \in \cap_{\eta \in \Omega_{2}} \Omega_{1, \eta}$ and $y_{0} \in \cap_{\xi \in \Omega_{1}} \Omega_{2, \xi}$.

For instance, quadratic domains of the form

$$
\left\{\sum_{i=1}^{N} a_{i}^{2} x_{i}^{2}+\sum_{j=1}^{M} b_{j}^{2} y_{j}^{2}<1\right\},
$$

with $a_{i}, b_{j}>0$ for all $i$ and $j$, give examples of convex domains with the invariance property. Another class of similar examples are given by $\Omega=$ $\left\{(x, y) \in \mathbb{R}^{N} \times \mathbb{R}^{M}: x \in \Omega_{1},-\phi_{j}(x)<y_{j}<\phi_{j}(x)\right\}$ where $\Omega_{1} \subset \mathbb{R}^{N}$ is a bounded smooth domain, $\phi_{j} \in C^{1}\left(\Omega_{1}\right) \cap C\left(\bar{\Omega}_{1}\right)$ are concave, positive in $\Omega_{1}$, vanish at $\partial \Omega_{1}$ and all of them achieve their maximum at the same point $x_{0} \in \Omega_{1}$. In this case the symmetry of $\Omega$ is achieved since $x_{0} \in \cap_{\eta \in \Omega_{2}} \Omega_{1, \eta}$ and $0 \in \mathbb{R}^{M}$ lies in $\cap_{\xi \in \Omega_{1}} \Omega_{2, \xi}$. Moreover, $\Omega$ is a convex domain.

Examples of non convex domains can also be constructed in the spirit of the last example. For instance, if $\Omega_{1} \subset \mathbb{R}^{N}$ is smooth and bounded, then regions in $\mathbb{R}^{N} \times \mathbb{R}$ of the form $\Omega=\left\{(x, y): x \in \Omega_{1}, \phi_{1}(x)<y<\phi_{2}(x)\right\}$ where $\phi_{i} \in C^{1}\left(\Omega_{1}\right) \cap C\left(\bar{\Omega}_{1}\right), \phi_{1}=\phi_{2}$ on $\partial \Omega_{1}$ and whose level sets $\phi_{1}>c$, $\phi_{2}<c^{\prime}$ are starshaped with respect to the same $x_{0} \in \Omega$ also satisfy the required invariance properties. An example of non convex domain of this type is described below. Let $\Gamma \subset \mathbb{R}^{2}$ be a closed smooth Jordan curve enclosing a domain $\Omega_{1},(0,0) \in \Omega_{1}$, and assume that $\Omega_{1}$ is starshaped with respect to $(0,0)$. Suppose $\varphi(\theta)=\rho(\theta) e^{i \theta}, \rho=|\varphi|, \theta=\operatorname{Arg} \varphi$, parameterizes $\Gamma$ and take a positive, decreasing function $h \in C^{1}[0,1]$ with $h^{\prime}(0)=h(1)=0$. Finally, define

$$
\phi(x, y)=h(t)
$$

where,

$$
t=\frac{|\zeta|}{|\varphi(\theta)|}, \quad \theta=\operatorname{Arg} \zeta, \quad \zeta=x+i y
$$

Then,

$$
\Omega=\left\{(x, y, z) \in \mathbb{R}^{3}:(x, y) \in \Omega_{1},-\phi(x, y)<z<\phi(x, y)\right\}
$$

provides the desired example (see Figure 1).

To conclude these remarks, it should be pointed out that the scaling invariance property $\Omega_{\lambda, \lambda^{\alpha / \beta}} \subset \Omega, 0<\lambda<1$, described above is a technical assumption to achieve uniqueness. By the moment, it is unclear to the authors if such uniqueness can be further shown in more general domains $\Omega$. In fact, the structure of the operator in (1.1) is, in several concepts, not compatible with the usual techniques employed for handling this issue in the more regular blow-up problems already studied in the literature. 

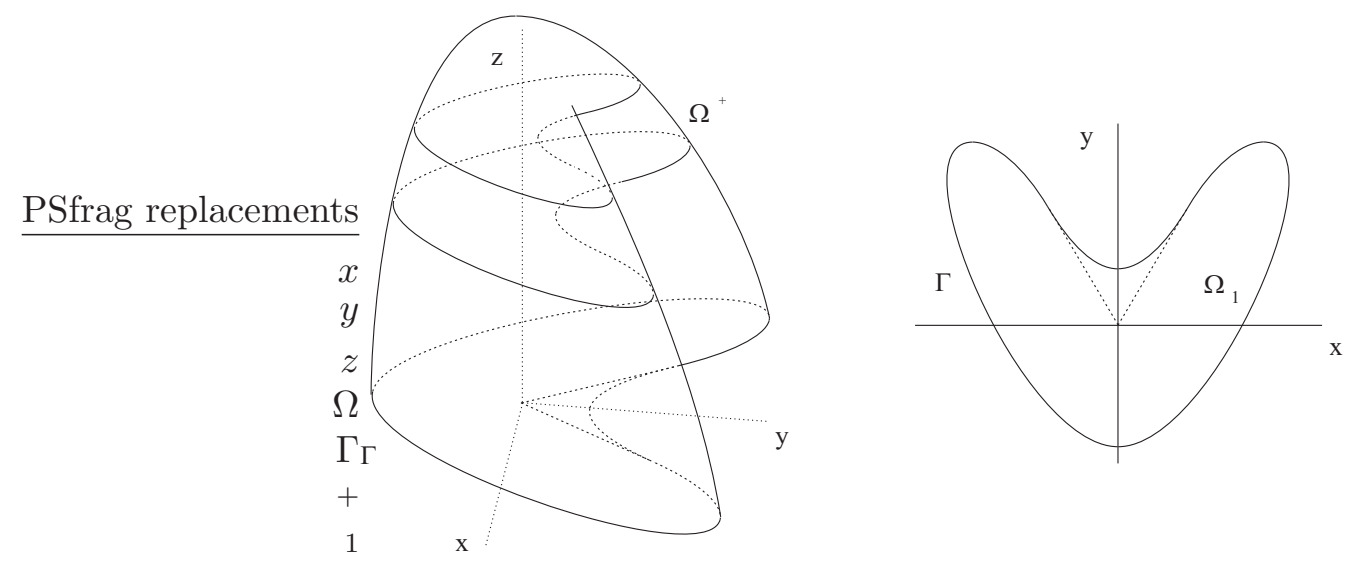

Figure 1. The half $\Omega^{+}$of a non convex domain in $\mathbb{R}^{3}$ with a starshaped section whose boundary is similar to the plane curve $\Gamma$.

\section{Boundary Behavior in General Domains}

In this final section we are dealing with the problem of finding the asymptotic profiles of positive solutions to (1.1) near the boundary. Recalling the results in Section 3 we need to distinguish between horizontal and vertical points on $\partial \Omega$. If $\nu(z)=\left(\nu_{1}(z), \nu_{2}(z)\right)$ is the outward unit normal at $z=(x, y) \in \partial \Omega, z_{0}=\left(x_{0}, y_{0}\right)$ is defined as a "horizontal" (respectively, "vertical") point if $\nu_{1}\left(z_{0}\right)=0\left(\right.$ resp. $\left.\nu_{2}\left(z_{0}\right)=0\right)$.

As we already pointed out in the introduction, we are showing that the variable $y$ controls the blow-up rates at non vertical points, since we are assuming $q>p$, and that the upper estimates at vertical points depends on the order of tangency of $\partial \Omega$ there.

Let us begin by making precise the notion of order of tangency in the $y$-direction. If $z_{0}=\left(x_{0}, y_{0}\right) \in \partial \Omega$ is a vertical point of $\partial \Omega$ and $\partial \Omega$ is locally characterized near $z_{0}$ by a smooth real function $\phi$ as $\phi(x, y)=0$ then necessarily

$$
\partial_{y_{i}} \phi\left(z_{0}\right)=0
$$

for all $i \in\{1, \ldots, M\}$. It is said that $z_{0}$ possesses an order of tangency $m \in \mathbb{N}$ in the $y$-direction if $\partial_{y}^{\gamma} \phi\left(z_{0}\right)=0$ for all multi indexes $\gamma \in \mathbb{N}^{M}$ such that $|\gamma| \leq m-1$ and $\partial_{y}^{\gamma} \phi\left(z_{0}\right) \neq 0$ for some $\gamma$ with $|\gamma|=m$. Such order of tangency is infinite if $\phi$ if of class $C^{\infty}$ near $z_{0}$ and partial derivatives $\partial_{y}^{\gamma} \phi\left(z_{0}\right)$ vanish for all $\gamma \in \mathbb{N}^{M}$. For instance, in the case of box domains $\Omega=\Omega_{1} \times \Omega_{2}$ considered in Section 3 all points on the part $\partial \Omega_{1} \times \Omega_{2}$ of the boundary are vertical with an infinite order of tangency (in the $y$-direction).

We can already state our first result providing an upper bound for the behavior of solutions near the boundary. We find that, while the order of magnitude of the growth of solutions when $z$ approaches horizontal points is that predicted by the term with $q$, namely $d^{-\beta}$ where

$$
\beta=\frac{q}{r-q+1},
$$


the situation is completely different when $z$ becomes close to vertical point.

Theorem 10. Assume $\Omega \subset \mathbb{R}^{N} \times \mathbb{R}^{M}$ is a smooth bounded domain and $r>q \geq p>1$. Let $u \in W_{\text {loc }}^{1, p, q}(\Omega) \cap L_{\text {loc }}^{\infty}(\Omega)$ be an arbitrary positive solution to (1.1). Then, there exists a constant $C$ depending only on $p, q$ and $\operatorname{diam}(\Omega)$ such that

$$
u(x, y) \leq C d(x, y)^{-\beta},
$$

for all $(x, y) \in \Omega$. If, in addition, $z_{0}=\left(x_{0}, y_{0}\right)$ is a vertical point in $\partial \Omega$ with an order $m$ of tangency in the $y$ direction, then

$$
\varlimsup
$$

when $z=(x, y)$ converges to $z_{0}$ following the inner normal at $z_{0}$ and the exponent $\gamma$ is given by

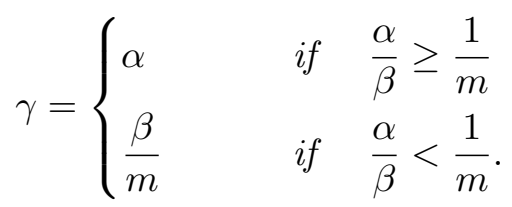

In particular,

$$
\varlimsup
$$

if $z_{0}$ possesses an infinite order of tangency in the $y$-direction.

Proof. To achieve the upper estimate (5.1) we proceed with a scaling argument. For $z=(x, y) \in \Omega$ set $d=d(x, y)$ and choose $\rho$ so that

$$
\rho^{2}+\rho^{\frac{2 \alpha}{\beta}}=d^{2} \text {. }
$$

Then

$$
B_{1}(x, \rho) \times B_{2}\left(y, \rho^{\alpha / \beta}\right) \subset B(z, d) \subset \Omega .
$$

Define

$$
\hat{v}(\xi, \eta)=\rho^{\alpha} u\left(x+\rho \xi, y+\rho^{\alpha / \beta} \eta\right) \quad(\xi, \eta) \in B_{1} \times B_{2},
$$

with $B_{1}, B_{2}$ the unit balls in $\mathbb{R}^{N}$ and $\mathbb{R}^{M}$, respectively. Then it is easily seen that $\hat{v}$ verifies the equation $\Delta_{p, q} \hat{v}=\hat{v}^{r}$ in $B_{1} \times B_{2}$, so that by comparison:

$$
\hat{v} \leq v
$$

in $B_{1} \times B_{2}$ where $v=v(\xi, \eta)$ is the unique solution to (1.1) in $B_{1} \times B_{2}$ (cf. Remarks 5 (a)). Therefore,

$$
u(x, y) \leq \rho^{-\alpha} v(0,0),
$$

and (5.1) follows by noticing that

$$
\rho^{\alpha} \leq d^{\beta} \leq C \rho^{\alpha} \quad(x, y) \in \Omega,
$$

where $C$ is a constant which depends only on $\alpha, \beta$ and the diameter of $\Omega$.

Assume now that $z_{0}$ is a vertical point of $\partial \Omega$. This means that the normal $\nu$ at $z_{0}$ has the form $\nu=\left(\nu_{1}, 0\right)$. Since problem (1.1) is translation invariant and also invariant with respect to rotations in $x$, we may assume without loss of generality that $z_{0}=0$ together with $\nu=\left(e_{1}, 0\right)$ with $e_{1}=(1, \ldots, 0) \in \mathbb{R}^{N}$. This amounts to saying that $\partial \Omega$ can be represented near $(0,0)$ as

$$
x_{1}=\phi\left(x^{\prime}, y\right) \quad\left(x^{\prime}, y\right) \in \mathbb{R}^{N-1} \times \mathbb{R}^{M},
$$


with a smooth function $\phi$ verifying $\phi(0,0)=0$ and $\nabla_{\left(x^{\prime}, y\right)} \phi(0,0)=0$, while $\Omega$ is additionally characterized as $x_{1}>\phi\left(x^{\prime}, y\right)$ when $\left(x^{\prime}, y\right)$ is close enough to $(0,0)$.

We now take $z=\left(\bar{x}_{1}, x^{\prime}, y\right) \in \Omega$ in the normal direction at $(0,0)$, i.e. $z=\left(\bar{x}_{1}, 0,0\right)$ with $\bar{x}_{1}>0$, and want to find $\rho=\rho\left(\bar{x}_{1}\right)$ such that

$$
B_{1}\left(\left(\bar{x}_{1}, 0\right), \rho\right) \times B_{2}\left(0, \rho^{\alpha / \beta}\right) \subset \Omega,
$$

as $\bar{x}_{1} \rightarrow 0$. Accordingly, set

$$
\rho=(1-\theta) \bar{x}_{1},
$$

with $0<\theta<1$. We first observe that $x_{1}>\bar{x}_{1}-\rho,\left|x^{\prime}\right|<\rho$ for $\left(x_{1}, x^{\prime}\right) \in$ $B_{1}\left(\left(\bar{x}_{1}, 0\right), \rho\right)$. On the other hand

$$
\left|\phi\left(x^{\prime}, y\right)\right| \leq C\left(\left|x^{\prime}\right|^{2}+|y|^{m}\right)
$$

for some $C>0$ and $\left(x^{\prime}, y\right)$ near $(0,0)$ (observe that (5.2) may be artificially regarded as the definition of order of tangency $m$ without increasing the smoothness degree of $\phi$ ). Now, the inequality

$$
\theta \bar{x}_{1}>C \rho^{2},
$$

holds as $\bar{x}_{1} \rightarrow 0$ provided $1>\theta \geq \theta_{0}>0$. Under this assumption

$$
x_{1}>\phi\left(x^{\prime}, 0\right)
$$

for $\left(x_{1}, x^{\prime}\right) \in B_{1}\left(\left(\bar{x}_{1}, 0\right), \rho\right)$. Thus,

$$
B_{1}\left(\left(\bar{x}_{1}, 0\right), \rho\right) \subset \Omega \cap\left\{z \in \mathbb{R}^{N} \times \mathbb{R}^{M}: y=0\right\} .
$$

By employing again $(5.2)$ we notice that $B_{1}\left(\left(\bar{x}_{1}, 0\right), \rho\right) \times B_{2}\left(0, \rho^{\alpha / \beta}\right) \subset \Omega$ provided

$$
\theta \bar{x}_{1}>C\left(\rho^{2}+\rho^{m \alpha / \beta}\right),
$$

holds true for small $\bar{x}_{1}$. Since $\theta \bar{x}_{1}=O(\rho)$, it suffices with having $\theta \bar{x}_{1}>$ $C \rho^{m \alpha / \beta}$ as $\bar{x}_{1} \rightarrow 0$.

Now, for $\alpha / \beta \geq 1 / m,(5.3)$ is satisfied for small $\bar{x}_{1}$ by taking, if necessary, a constant $\theta$, close enough to 1 when $\alpha / \beta$ is $1 / m$.

On the other hand, when $\alpha / \beta<1 / m$ we take

$$
1-\theta=k \bar{x}_{1}^{\gamma},
$$

with $\gamma>0$ and $k$ a positive constant. Then, (5.3) is achieved if $\gamma$ is chosen so that

$$
\gamma=\frac{1}{m}-\frac{\alpha}{\beta},
$$

and $k$ is small enough. Since $\theta \rightarrow 1$ under this choice we have again the desired inclusion $B_{1}\left(\left(\bar{x}_{1}, 0\right), \rho\right) \times B_{2}\left(0, \rho^{\alpha / \beta}\right) \subset \Omega$ with the above election for $\theta$ and $\rho$.

Finally, by arguing as in the first part of the proof we get that

$$
u\left(x_{1}, 0,0\right) \leq \rho^{-\alpha} v((0,0), 0),
$$

for all conveniently small $x_{1}>0$. In case $\alpha / \beta \geq 1 / m, \rho \sim(1-\theta) x_{1}$ with $\theta \in(0,1)$ constant. Thus, $u\left(x_{1}, 0,0\right) \leq C x_{1}^{-\alpha}$ as $x_{1} \rightarrow 0$. Observe in addition that provided the order of tangency of $\partial \Omega$ at $z_{0}$ (in the $y$-direction) 
is infinity then $m$ can always be chosen so that $\alpha / \beta>1 / m$ and thus we get the above estimate.

If, on the other hand, $\alpha / \beta<1 / m$ then

$$
\rho \sim k_{1} x_{1}^{\gamma+1} \quad x_{1} \rightarrow 0+,
$$

with $k_{1}>0$ a constant, and so $u\left(x_{1}, 0,0\right) \leq C x_{1}^{-\beta / m}$ for $x_{1}$ near zero.

The proof is completed by observing that $d(x, y)=x_{1}$ on points on the normal direction close enough to $(0,0)$.

Remark 8. The analysis of Section 3 reveals that in case of "flat" vertical portions of $\partial \Omega$, as in the example $\partial \Omega_{1} \times \Omega_{2} \subset \partial\left(\Omega_{1} \times \Omega_{2}\right)$ the exact upper order $\alpha$ of divergence of solutions to infinity is attained.

On the other hand, the case of the corner point $(0,0)$ in $\mathbb{R}_{+}^{2} \times \mathbb{R}_{+}^{2}$ reflects -in some sense- the opposite configuration to flatness. Indeed, as was shown at the end of Section 3 , the solution $u$ exhibits at those points all orders of divergence to infinity, ranging in the gap from $\alpha$ to $\beta$.

Next we prove complementary lower estimates of the asymptotics of solutions near the boundary. Now we restrict ourselves to consider $\Omega$ convex.

Theorem 11. Assume $\Omega \subset \mathbb{R}^{N} \times \mathbb{R}^{M}$ is a smooth, bounded and convex domain, and $0<p-1 \leq q-1<r$. Let $u \in W_{l o c}^{1, p, q}(\Omega) \cap L_{l o c}^{\infty}(\Omega)$ be an arbitrary positive solution to (1.1). Then, the following properties hold.

a) If $z_{0}=\left(x_{0}, y_{0}\right) \in \partial \Omega$ is not a vertical point then

$$
\varliminf_{(x, y) \rightarrow\left(x_{0}, y_{0}\right)} d(x, y)^{\beta} u(x, y) \geq\left|\nu_{2}\left(z_{0}\right)\right|^{\beta} A_{q},
$$

where $\nu\left(z_{0}\right)=\left(\nu_{1}\left(z_{0}\right), \nu_{2}\left(z_{0}\right)\right)$ is the outward unit normal at $z_{0}$ and $A=A_{q}$ verifies $A^{r-q+1}=(q-1) \beta^{q-1}(\beta+1)$.

b) If $z_{0}$ is a vertical point then

$$
\varliminf_{(x, y) \rightarrow\left(x_{0}, y_{0}\right)} d(x, y)^{\alpha} u(x, y) \geq A_{p},
$$

where $z \rightarrow z_{0}$ along the inner normal at $z_{0}$ and $A=A_{p}$ verifies $A^{r-p+1}=$ $(p-1) \alpha^{p-1}(\alpha+1)$.

Proof. For $z_{0} \in \partial \Omega$ it follows from the convexity of $\Omega$ that

$$
-\nu\left(z_{0}\right)\left(z-z_{0}\right)>0
$$

for all $z \in \Omega$. We are looking for solutions to the equation $\Delta_{p, q} u=u^{r}$ of the form,

in $t>0$.

$$
u(x, y)=w(t) \quad t=:-\nu\left(z_{0}\right)\left(z-z_{0}\right)>0,
$$

Smooth solutions of that type must satisfy the ordinary differential equation

$$
\left|\nu_{1}\left(z_{0}\right)\right|^{p}\left(\left|w^{\prime}\right|^{p-2} w^{\prime}\right)^{\prime}+\left|\nu_{2}\left(z_{0}\right)\right|^{q}\left(\left|w^{\prime}\right|^{q-2} w^{\prime}\right)^{\prime}=w^{r} .
$$


We claim the existence of a unique positive solution $w \in C^{2}(0,+\infty)$ to (5.6) such that

$$
w(0+)=\infty .
$$

Such solution satisfies in addition

$$
\lim _{t \rightarrow \infty} w(t)=\lim _{t \rightarrow \infty} w^{\prime}(t)=0,
$$

together with

$$
w(t) \sim\left|\nu_{2}\left(z_{0}\right)\right|^{\beta} A_{q} t^{-\beta},
$$

as $t \rightarrow 0+$. We will use the notation $w=w\left(t, z_{0}\right)$ in what follows.

We now proceed to show the claim. As a first remark, the expression

$$
\frac{\left|\nu_{1}\left(z_{0}\right)\right|^{p}}{p^{\prime}}\left|w^{\prime}\right|^{p}+\frac{\left|\nu_{2}\left(z_{0}\right)\right|^{q}}{q^{\prime}}\left|w^{\prime}\right|^{q}-\frac{w^{r+1}}{r+1}
$$

is constant on any smooth solution $w$ to (5.6).

On the other hand, every positive solution to (5.6) which is defined in the whole of $(0,+\infty)$ and fulfills (5.7) must have $w^{\prime}(t)<0$ for all $t>0$. In fact, define

$$
F(s)=\frac{\left|\nu_{1}\left(z_{0}\right)\right|^{p}}{p^{\prime}} s^{p}+\frac{\left|\nu_{2}\left(z_{0}\right)\right|^{q}}{q^{\prime}} s^{q},
$$

in $s \geq 0$, and $G(s)=F^{-1}(s)$. Then, any solution having $w\left(t_{0}\right)=w_{0}>0$ together with $w^{\prime}\left(t_{0}\right)=0$ for $t_{0}>0$ makes the integral

$$
\int_{w_{0}}^{\infty} \frac{d s}{G\left(\frac{s^{r+1}}{r+1}-\frac{w_{0}^{r+1}}{r+1}\right)}
$$

finite. In that case, $w$ can not be continued to the whole of $(0,+\infty)$.

With this kind of ideas it can be checked that our positive solution $w$, defined in $(0,+\infty)$ and satisfying (5.7) also satisfies

$$
\lim _{t \rightarrow \infty} w(t)=\lim _{t \rightarrow \infty} w^{\prime}(t)=0 .
$$

Thus, $w$ is implicitly given by the expression

$$
\int_{w}^{\infty} \frac{d s}{G\left(\frac{s^{r+1}}{r+1}\right)}=t
$$

Since

as $u \rightarrow \infty$ then it follows that

$$
G(u) \sim \frac{\left(q^{\prime} u\right)^{1 / q}}{\left|\nu_{2}\left(z_{0}\right)\right|}
$$

$$
w(t)=\left|\nu_{2}\left(z_{0}\right)\right|^{\beta} \beta^{\beta}\left(\frac{r+1}{q^{\prime}}\right)^{\beta / q} t^{-\beta}(1+o(1))=\left|\nu_{2}\left(z_{0}\right)\right|^{\beta} A_{q} t^{-\beta}(1+o(1)),
$$

as $t \rightarrow 0+$. This completes the proof of the claim.

Let us proceed now to show (5.4) and suppose $z_{n} \rightarrow z_{0}$. If $\bar{z}_{n}$ stands for the closest point to $z_{n}$ on $\partial \Omega$ then $\bar{z}_{n} \rightarrow z_{0}$ and for any $\delta>0, \bar{z}_{n} \in \mathcal{U}_{\delta}=$ $B\left(z_{0}, \delta\right) \cap \partial \Omega$ for large $n$.

On the other hand, by comparison

$$
d\left(z_{n}\right)^{\beta} u\left(z_{n}\right) \geq t^{\beta} w\left(t, \bar{z}_{n}\right),
$$


with $t=d\left(z_{n}\right)$ where $w\left(t, \bar{z}_{n}\right)$ designates the solution to $(5.6)-$ with $z_{0}$ replaced by $\bar{z}_{n}$ - satisfying (5.7), and defined in $(0,+\infty)$. Now we want to obtain uniform estimates in $n$ of $t^{\beta} w\left(t, \bar{z}_{n}\right)$ when $0<t<\varepsilon$ and $\varepsilon>0$ is small enough. Setting, $\theta_{\delta}^{+}=\max _{\mathcal{U}_{\delta}}\left|\nu_{2}\right|, \theta_{\delta}^{-}=\min _{\mathcal{U}_{\delta}}\left|\nu_{2}\right|, w_{n}(t)=w\left(t, \bar{z}_{n}\right)$,

$$
F_{1}(s)=\frac{\left(1-\left(\theta_{\delta}^{+}\right)^{2}\right)^{p / 2}}{p^{\prime}} s^{p}+\frac{\left(\theta_{\delta}^{-}\right)^{q}}{q^{\prime}} s^{q}
$$

and $G_{1}(s)=F_{1}^{-1}(s)$ we find

$$
\frac{w_{n}^{r+1}}{r+1}=F\left(\left|w_{n}^{\prime}\right|, \bar{z}_{n}\right) \geq F_{1}\left(\left|w_{n}^{\prime}\right|\right)
$$

so that

$$
\int_{w_{n}(t)}^{\infty} \frac{d s}{G_{1}\left(\frac{s^{r+1}}{r+1}\right)} \leq t
$$

This inequality entails that for an arbitrary $M>0$ we have $w_{n}(t)>M$ uniformly in $n$ provided $0<t<\varepsilon$ for a certain $\varepsilon>0$ only depending on $M$. In particular, $\lim w_{n}\left(t_{n}\right)=\infty$ if $t_{n} \rightarrow 0$.

A further consequence of $(5.9)$ is the existence of a function $h_{\delta}(w)$ with $\lim _{w \rightarrow \infty} h_{\delta}(w)=0$, such that,

$$
\left(1+h_{\delta}\left(w_{n}(t)\right) \int_{w_{n}(t)}^{\infty} \frac{d s}{s^{\frac{r+1}{q}}} \leq\left(\theta_{\delta}^{-}\right)^{-1}\left(\frac{r+1}{q^{\prime}}\right)^{-1 / q} .\right.
$$

It readily follows from this relation that

$$
t^{\beta} w_{n}(t) \geq\left(\theta_{\delta}^{-}\right)^{\beta} \beta^{\beta}\left(\frac{r+1}{q^{\prime}}\right)^{\beta / q}\left(1+h_{\delta}\left(w_{n}(t)\right)^{\beta} .\right.
$$

Setting $t=t_{n}$, letting $t_{n} \rightarrow 0$ and recalling that $\lim w_{n}\left(t_{n}\right)=\infty$ we get

$$
\underline{\lim } t_{n}^{\beta} w_{n}\left(t_{n}\right) \geq\left(\theta_{\delta}^{-}\right)^{\beta} A_{q},
$$

for all small $\delta>0$. Further letting $\delta \rightarrow 0+$ we find

$$
\underline{\lim } t_{n}^{\beta} w_{n}\left(t_{n}\right) \geq\left|\nu_{2}\left(z_{0}\right)\right|^{\beta} A_{q} .
$$

Via (5.8) we finally conclude,

$$
\underline{\lim } d_{n}^{\beta} u\left(z_{n}\right) \geq\left|\nu_{2}\left(z_{0}\right)\right|^{\beta} A_{q} .
$$

with $d_{n}=d\left(z_{n}\right)$, as desired.

To finish the proof of the theorem, we observe that the estimate (5.5) is a direct consequence of the inequality

$$
u(x, y) \geq w\left(t, z_{0}\right),
$$

$t=-\nu\left(z_{0}\right)\left(z-z_{0}\right)$, where, being now $z_{0}$ a vertical point, the coefficient $\left|\nu_{2}\left(z_{0}\right)\right|^{q}$ vanishes in equation (5.6).

Remark 9. If $z_{0} \in \partial \Omega$ and for $0<\theta<1, K_{\theta}$ designates the conical region $K_{\theta}=\left\{z \in \Omega:\left\langle z-z_{0},-\nu\left(z_{0}\right)\right\rangle \geq \theta\left|z-z_{0}\right|\right\}$, then it can be checked that

$$
d(z) \sim t(z)=-\left\langle z-z_{0}, \nu\left(z_{0}\right)\right\rangle,
$$


as $z \rightarrow z_{0}$. Therefore, (5.5) can be written as,

$$
\varliminf_{z \rightarrow z_{0}, z \in K_{\theta}} d(z)^{\alpha} u(z) \geq A_{p}
$$

Acknowledgements. Supported by Spanish Ministerio de Ciencia e Innovacion under grant MTM2008-05824. J. D. Rossi is partially supported by UBA X066 and by CONICET, Argentina.

\section{REFERENCES}

[1] S. Antontsev, S. Shmarev, Elliptic Equations with Anisotropic Nonlinearity and Nonstandard Growth Conditions, in "Handbook of Differential Equations: Stationary Partial Differential Equations", Vol. 3 (M. Chipot and P. Quittner, Editors) (2006), 1-100.

[2] C. Bandle, M. Marcus, 'Large' solutions of semilinear elliptic equations: Existence, uniqueness and asymptotic behaviour, J. Anal. Math. 58 (1992), 9-24.

[3] M. Bendahmane, M. Langlais, M. SaAd, On some anisotropic reaction-diffusion systems with $L^{1}$-data modeling the propagation of an epidemic disease, Nonlinear Anal. 54 (2003), 617-636

[4] M. Bendahmane, K. H. Karlsen, Renormalized solutions of an anisotropic reaction-diffusion-advection system with $L^{1}$ data, Commun. Pure Appl. Anal. 5 (2006), 733-762.

[5] L. Bieberbach, $\Delta u=e^{u}$ und die automorphen Funktionen, Math. Ann. 77 (1916), $173-212$.

[6] M. Chuaqui, C. Cortázar, M. Elgueta, J. García-Melián, Uniqueness and boundary behaviour of large solutions to elliptic problems with singular weights, Comm. Pure Appl. Anal. 3 (2004), 653-662.

[7] M. Del Pino, R. Letelier, The influence of domain geometry in boundary blow-up elliptic problems, Nonlinear Anal. 48 (6) (2002), 897-904.

[8] G. DíAz, R. LETELIER, Explosive solutions of quasilinear elliptic equations: Existence and uniqueness, Nonlinear Anal. 20 (1993), 97-125.

[9] A. El Hamidi, J.M. Rakotoson. Extremal functions for the anisotropic Sobolev inequalities, Ann. Institut H. Poincaré AN 24 (2007), 741-756.

[10] A. El Hamidi, J. VÉtois, Sharp Sobolev asymptotics for critical anisotropic equations, Arch. Rat. Mech. Anal. 192 (2009), 1-36.

[11] I. Fragala, F. Gazzola AND B. KAWOHL, Existence and nonexistence results for anisotropic quasilinear elliptic equation, Ann. Inst. H. Poincaré AN 21 (2004), 715-734.

[12] J. García-Melián, Nondegeneracy and uniqueness for boundary blow-up elliptic problems, J. Diff. Eqns. 223 (2006), 208-227.

[13] J. García Melián, Quasilinear equations with boundary blow-up and exponential reaction, Adv. Nonl. Stud. 9 (2009), 149-160.

[14] J. García Melián, Large solutions for equations involving the p-Laplacian and singular weights. Z. Angew. Math. Phys. 60 (2009), 594-607.

[15] J. García-Melián, R. Letelier-Albornoz, J. Sabina de Lis, Uniqueness and asymptotic behaviour for solutions of semilinear problems with boundary blow-up, Proc. Amer. Math. Soc. 129 (12) (2001), 3593-3602.

[16] M. Giaquinta, A counter-example to the boundary regularity of solutions to elliptic quasilinear systems, Manuscripta Math. 24 (1978), no. 2, 217-220.

[17] D. Gilbarg, N.S. Trudinger, Elliptic partial differential equations of second order, Springer-Verlag, 1983.

[18] J. B. Keller, On solutions of $\Delta u=f(u)$, Comm. Pure Appl. Math. 10 (1957), $503-510$.

[19] O. A. Ladyzhenskaya, N. N. URAltseva, Linear and Quasilinear Elliptic Equations, Academic Press, New York, 1968. 
[20] A. C. LAzer, P. J. MCKenna, On a problem of Bieberbach and Rademacher, Nonlinear Anal. 21 (1993), 327-335.

[21] G. Lieberman, Boundary regularity for solutions of degenerate elliptic equations, Nonlinear Anal. 12 (1988), 1203-1219.

[22] G. Lieberman, Gradient estimates for a new class of degenerate elliptic and parabolic equations, Ann. Scuola Norm. Sup. Pisa Cl. Sci. (4) 21 (1994), no. 4, 497-522.

[23] C. Loewner, L. Nirenberg, Partial differential equations invariant under conformal or projective transformations, in "Contributions to Analysis (a collection of papers dedicated to Lipman Bers)", Academic Press, New York, 1974, p. 245-272.

[24] P. Marcellini, Regularity of minimizers of integrals of the calculus of variations with nonstandard growth conditions, Arch. Rat. Mech. Anal. 105 (1989), 267-284.

[25] J. MAtero, Quasilinear elliptic equations with boundary blow-up, J. Anal. Math. 69 (1996), 229-247.

[26] R. Osserman, On the inequality $\Delta u \geq f(u)$, Pacific J. Math. 7 (1957), 1641-1647.

[27] H. RAdEMACHeR, Einige besondere Probleme partieller Differentialgleichungen, in "Die Differential- und Integralgleichungen der Mechanik und Physik I", 2nd. ed. (P. Frank, R. von Mises, Editors), Rosenberg, New York (1943), 838-845.

[28] V. RĂDUlEsCU, Singular phenomena in nonlinear elliptic problems: from boundary blow-up solutions to equations with singular nonlinearities, in "Handbook of Differential Equations: Stationary Partial Differential Equations", Vol. 4 (M. Chipot, Editor) (2007), 483-591.

[29] J. RÁKosník, Some remarks to anisotropic Sobolev spaces I, Beiträge Anal. 13 (1979), 55-68.

[30] J. RÁkosník, Some remarks to anisotropic Sobolev spaces II, Beiträge Anal. 15 (1980), 127-140.

[31] M. Struwe, Variational methods. Applications to nonlinear partial differential equations and Hamiltonian systems, Springer-Verlag, Berlin, 2008.

[32] A. S. Tersenov, A. S. Tersenov, The problem of Dirichlet for anisotropic quasilinear degenerate elliptic equations, J. Diff. Eqns. 235 (2007), 376-396.

[33] P. TOLKSDORF, Regularity for a more general class of quasilinear elliptic equations, J. Diff. Eqns. 51 (1984), no. 1, 126-150.

[34] J. VÉToIs, A priori estimates for solutions of anisotropic elliptic equations, Nonl. Anal. 71 (2009), no. 9, 3881-3905.

J. García-Melián and J. C. Sabina de Lis Departamento de Análisis Matemático, Universidad de La Laguna. C/. Astrofísico Francisco SÁnchez s/n, 38271 - La Laguna, SPAiN and

Instituto Universitario de Estudios Avanzados (IUdEA) en Física Atomica, Molecular y Fotonica, Facultad de Física, Universidad de La Laguna

C/. Astrofísico Francisco Sánchez s/n, 38203 - La Laguna , SPAin

E-mail address: jjgarmel@ull.es, josabina@ull.es

J. D. Rossi

Departamento de Matemática, FCEyN UBA,

Ciudad Universitaria, Pab 1, (1428),

Buenos Aires, Argentina.

E-mail address: jrossi@dm.uba.ar 